Research Article

\title{
Synchronous Stability of Four Homodromy Vibrators in a Vibrating System with Double Resonant Types
}

\author{
Xueliang Zhang $\mathbb{D D}^{1,2}$ Chao Li, ${ }^{1}$ Zhihui Wang, ${ }^{1}$ and Shiju Cui ${ }^{1}$ \\ ${ }^{1}$ School of Mechanical Engineering and Automation, Northeastern University, Shenyang, Liaoning 110819, China \\ ${ }^{2}$ State Key Laboratory of Tunnel Boring Machine, Northern Heavy Industries Group, Shenyang, Liaoning 110141, China \\ Correspondence should be addressed to Xueliang Zhang; luckyzxl7788@163.com
}

Received 12 July 2018; Accepted 31 October 2018; Published 4 December 2018

Academic Editor: Laurent Mevel

Copyright (C) 2018 Xueliang Zhang et al. This is an open access article distributed under the Creative Commons Attribution License, which permits unrestricted use, distribution, and reproduction in any medium, provided the original work is properly cited.

\begin{abstract}
This paper aims at studying the synchronous stability of four homodromy vibrators in subresonant and superresonant states. The motion differential equations are established firstly. The simplified form of analytical expressions is yielded, and the stability criterion of synchronous states satisfies Routh-Hurwitz criterion. The coupling dynamic characteristics of the system are analyzed in detail numerically, such as the maximum of coupling torque, coefficients of ability of synchronization and stability, and phase differences. Based on the ratio of operating frequencies to natural frequencies of the system, the resonant regions are divided into two areas: subresonant and superresonant. It is shown that the phase differences among four vibrators in the subresonant state are stabilized about zero, and exciting forces of four vibrators are positively superposed, while in the superresonant one, the phenomenon of the diversity of the nonlinear system occurs, i.e., two groups of synchronous and stable solutions of the phase differences (pi and pi/2) are found, and in this case, the exciting forces of four vibrators are counteracted, the rigid frame embodies no vibration, and the minimum of dynamic load transferring to foundation is realized. The correctness of theoretical results is verified by numerical characteristic analysis and simulations. This paper can provide a theoretical reference for designing a type of new high-frequency vibrating mill.
\end{abstract}

\section{Introduction}

Synchronization is a natural phenomenon and often occurs in daily life. For example, two organ pipes close to each other can produce synchronous sounds [1], synchronization phenomenon is observed in nonlinear circuits [2], and so on. The earliest scientific research on synchronization was given by Huygens [3], which attracted more and more researchers to engage in research on it. In 1990, Pecora studied synchronization in chaotic systems and proposed its application of communication security [4]. Osipov et al. introduced synchronization in networks of Oscillatory [5]. Teufel et al. and Kapitaniak et al. investigated synchronization of pendula $[6,7]$. Scholars Lu et al. analyzed synchronization on neuronal electrical activities [8]. Speed synchronization with the new hybrid system being used to dynamic shift coordinated control was given by Yan et al. [9]. With the rapid development of science and technology, the synchronization problems in other fields are also investigated by many scholars [10-13].

Vibration phenomena are also often encountered in science and have a great influence in engineering. For a long time, some scholars found synchronization in the field of vibration. In 1960s, theoretical research studies about synchronization of two vibrators were given firstly by Blekhman by using the method of direct separation of motions [14-16]. In 1980, Inoue and Araki studied triple frequency synchronization of vibrators driven by double motors [17]. Scholar Quinn et al. described the behavior of synchronization of resonance in a weakly coupled multidegree-of-freedom system [18]. Wen et al. investigated numerous types of synchronization problems of double 
identical vibrators and proposed the concept of vibratory synchronization transmission $[19,20]$. In modern times, many theories on synchronization of vibrators were also studied by other scholars, for example, Fang and Hou investigated synchronization characteristics of a rotorpendula system [21], and some research studies on selfsynchronization of nonideal sources or exciters were given by Balthazar et al. [22, 23]. Zhang et al. [24-26] and Zhao et al. [27], studied problems of vibratory synchronization transmission on a cylindrical roller and synchronization of two or three vibrators in a nonresonant vibrating system.

The above research studies are mainly devoted to synchronization problems of double or multiple vibrators in superresonant region, while synchronous and stable states of the subresonant region are mentioned rarely. This paper focuses on studying synchronous stability of four vibrators in the different resonant states (subresonant and superresonant). The dynamical model consists of four vibrators and a rigid frame. The vibrator is a kind of mechanism which generates exciting forces through eccentric rotors driven by AC motor. The present model is not complicated in structure, but the research results on synchronous and stable states of four vibrators are of great significance to engineering application.

In this paper, the figure of the model is given, and the motion differential equations are established in Section 2; the synchronization criterion of the four vibrators and the stability criterion are obtained in Section 3; characteristic analysis is discussed numerically and resonant regions are separated in Section 4; computer simulations are analyzed in Section 5; finally, conclusion is drawn.

\section{Dynamic Model Description}

In Figure 1(a), the dynamical model consisting of four vibrators and a rigid frame is given, thereof a vibrator is defined as eccentric rotor driven by an induction motor, which can motivate a certain exciting force to make the rigid frame vibrate with a certain type of movement. Four vibrators which rotate in the same directions are driven separately by four induction motors and are fixed on a rigid frame. The rigid frame and a foundation are connected by four springs. Figure 1(b) illustrates three reference coordinates of the vibrating system, which includes the fixed frame $o x y$; the frame $o^{\prime} x^{\prime} y^{\prime}$ is parallel to $o x y$, and the frame $o^{\prime \prime} x^{\prime \prime} y^{\prime \prime}$ rotates around $o^{\prime}$. The displacement vectors of the system in $x$ and $y$ directions are denoted by $x$ and $y$, respectively. The swing angle of the rigid frame is $\psi$. The rotational angles of four eccentric rotors are denoted by $\varphi_{i}, i=1,2,3,4$. The angles between four vibrators and the $x$-axis are $\beta_{1}, \beta_{2}, \beta_{3}$, $\beta_{4}$, respectively, with $\beta_{1}=0^{\circ}, \beta_{2}=90^{\circ}, \beta_{3}=180^{\circ}$, and $\beta_{4}=270^{\circ}$.

Based on Lagrange's equations and referring to [26], the motion differential equations of the vibrating system are established as the following form:

$$
\begin{aligned}
M \ddot{x}+f_{x} \dot{x}+k_{x} x= & \sum_{i=1}^{4} m_{i} r\left(\dot{\varphi}_{i}^{2} \cos \varphi_{i}+\ddot{\varphi}_{i} \sin \varphi_{i}\right), \\
M \ddot{y}+f_{y} \dot{y}+k_{y} y= & \sum_{i=1}^{4} m_{i} r\left(\dot{\varphi}_{i}^{2} \sin \varphi_{i}-\ddot{\varphi}_{i} \cos \varphi_{i}\right), \\
J \ddot{\psi}+f_{\psi} \dot{\psi}+k_{\psi} \psi= & l_{\mathrm{o}} r \sum_{i=1}^{4} m_{i}\left[\dot{\varphi}_{i}^{2} \sin \left(\varphi_{i}-\beta_{i}\right)\right. \\
& \left.-\ddot{\varphi}_{i} \cos \left(\varphi_{i}-\beta_{i}\right)\right], \\
\left(J_{\mathrm{o} i}+m_{i} r^{2}\right) \ddot{\varphi}_{i}+f_{i} \dot{\varphi}_{i}= & T_{\mathrm{e} i}-m_{i} r\left[\ddot{y} \cos \varphi_{i}-\ddot{x} \sin \varphi_{i}\right. \\
& +l_{0} \ddot{\psi} \cos \left(\varphi_{i}-\beta_{i}\right) \\
& \left.+l_{0} \dot{\psi}^{2} \sin \left(\varphi_{i}-\beta_{i}\right)\right], \quad i=1,2,3,4,
\end{aligned}
$$

where $M=m+m_{1}+m_{2}+m_{3}+m_{4}, f_{\psi}=\left(f_{x} l_{y}^{2}+f_{y} l_{x}^{2}\right) / 2$, $J=J_{\mathrm{m}}+\left(m_{1}+m_{2}+m_{3}+m_{4}\right) l_{0}^{2}, J=M l_{\mathrm{e}}^{2}$, and $k_{\psi}=\left(k_{x} l_{y}^{2}+\right.$ $\left.k_{y} l_{x}^{2}\right) / 2$.

Here $m$ is the mass of the rigid frame; $m_{i}$ is the mass of the exciter $i, i=1,2,3,4 ; M$ is the total mass of the vibrating system, which includes the mass of the rigid frame $m$ and the mass of the four exciters; $f_{i}$ is damping coefficients of the axes of induction motor $i, i=1,2,3,4 ; f_{x}, f_{y}, f_{\psi}$ are damping constants of the vibrating system in $x, y$, and $\psi$ directions, respectively; $J$ is the moment of inertia of the vibrating system about its mass center and $J_{\mathrm{m}}$ is that of the rigid frame about its mass center; $l_{\mathrm{e}}$ is equivalent rotational radius of the vibrating system about the mass center of the rigid frame; $l_{0}$ denotes the distance between the rotational center of the exciter $i$ and the mass center of the rigid frame; $k_{x}, k_{y}, k_{\psi}$ are respectively stiffnesses of the vibrating system in $x, y$, and $\psi$ directions; $T_{\mathrm{e} i}$ [27] is electromagnetic torque of the motor $i, i=1,2,3,4$; and $r$ is the eccentric radius of the exciter.

\section{Theoretical Derivation}

It is assumed that the average phase of four vibrators is $\varphi$; the phase differences between vibrators 1 and 2, 2 and 3 , and 3 and 4 are $2 \alpha_{1}, 2 \alpha_{2}$, and $2 \alpha_{3}$, respectively, that is,

$$
\begin{aligned}
\varphi & =\frac{1}{4} \sum_{i=1}^{4} \varphi_{i}, \\
\varphi_{1}-\varphi_{2} & =2 \alpha_{1}, \\
\varphi_{2}-\varphi_{3} & =2 \alpha_{2}, \\
\varphi_{3}-\varphi_{4} & =2 \alpha_{3} .
\end{aligned}
$$

The change of average angular velocity $\dot{\varphi}$ of four exciters is periodic because the vibrating system vibrates regularly. We can assume that the least common multiple period of four exciters is $T$, the average value $\omega_{\mathrm{m} 0}$ of the average angular velocity $\dot{\varphi}$ during time $T$ must be a constant, i.e., $\omega_{\mathrm{m} 0}=(1 / T) \int_{t}^{t+T} \dot{\varphi} d t=$ constant. $\omega_{\mathrm{m} 0}$ denotes the average angular velocity of four exciters and is also referred to as the synchronous operating frequency in the steady state. 


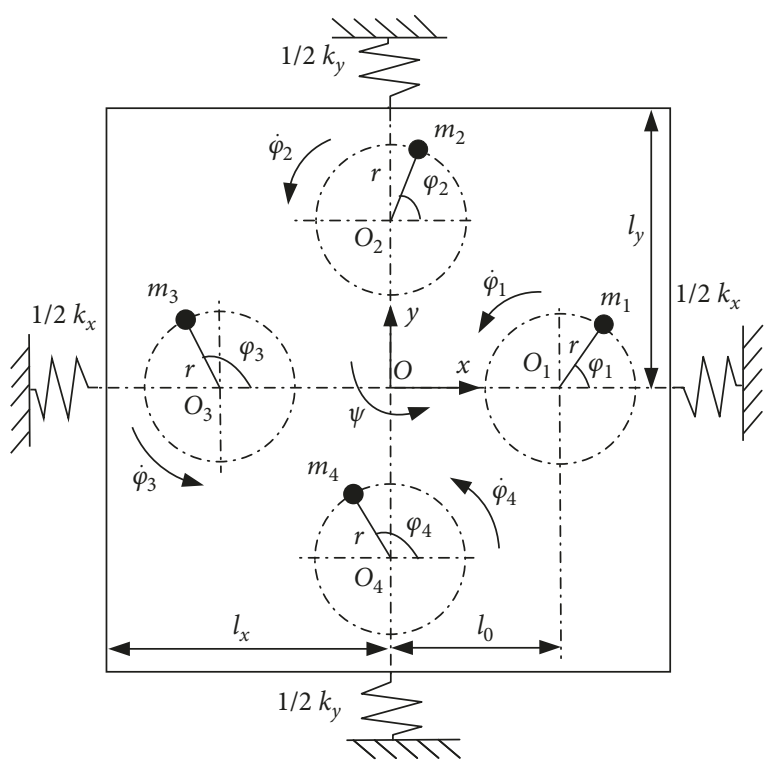

(a)

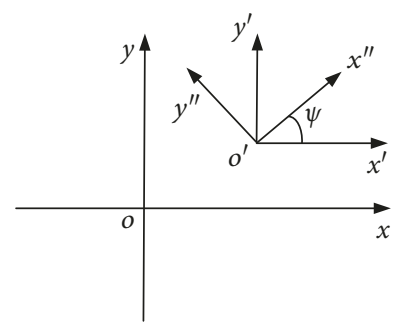

FIGURE 1: Model and coordinate systems. (a) Dynamic model of the vibrating system; (b) three reference coordinate systems.

According to the modified average method of small parameters $[25,26]$, we can introduce small parameters $\varsigma_{0}, \varsigma_{i}$ $\left(\varsigma_{0}, \varsigma_{i}\right.$ are functions of time, $\left.i=1,2,3\right)$. It is assumed that the coefficients of the instantaneous change of $\dot{\varphi}$ around $\omega_{\mathrm{m} 0}$, i.e., $\dot{\varphi}=\left(1+\varsigma_{0}\right) \omega_{\mathrm{m} 0}, \dot{\alpha}_{i}=\varsigma_{i} \omega_{\mathrm{m} 0}, i=1,2,3$.

Thus, the rearrangement of Equation (2) is expressed as

$$
\begin{aligned}
& \varphi_{1}=\varphi+\frac{3}{2} \alpha_{1}+\alpha_{2}+\frac{1}{2} \alpha_{3}=\varphi+v_{1}, \\
& \varphi_{2}=\varphi-\frac{1}{2} \alpha_{1}+\alpha_{2}+\frac{1}{2} \alpha_{3}=\varphi+v_{2}, \\
& \varphi_{3}=\varphi-\frac{1}{2} \alpha_{1}-\alpha_{2}+\frac{1}{2} \alpha_{3}=\varphi+v_{3}, \\
& \varphi_{4}=\varphi-\frac{1}{2} \alpha_{1}-\alpha_{2}-\frac{3}{2} \alpha_{3}=\varphi+v_{4}, \\
& \dot{\varphi}_{1}=\left(1+\varsigma_{0}+\frac{3}{2} \varsigma_{1}+\varsigma_{2}+\frac{1}{2} \varsigma_{3}\right) \omega_{\mathrm{m} 0}=\left(1+\varepsilon_{1}\right) \omega_{\mathrm{m} 0}, \\
& \dot{\varphi}_{2}=\left(1+\varsigma_{0}-\frac{1}{2} \varsigma_{1}+\varsigma_{2}+\frac{1}{2} \varsigma_{3}\right) \omega_{\mathrm{m} 0}=\left(1+\varepsilon_{2}\right) \omega_{\mathrm{m} 0}, \\
& \dot{\varphi}_{3}=\left(1+\varsigma_{0}-\frac{1}{2} \varsigma_{1}-\varsigma_{2}+\frac{1}{2} \varsigma_{3}\right) \omega_{\mathrm{m} 0}=\left(1+\varepsilon_{3}\right) \omega_{\mathrm{m} 0}, \\
& \dot{\varphi}_{4}=\left(1+\varsigma_{0}-\frac{1}{2} \varsigma_{1}-\varsigma_{2}-\frac{3}{2} \varsigma_{3}\right) \omega_{\mathrm{m} 0}=\left(1+\varepsilon_{4}\right) \omega_{\mathrm{m} 0}, \\
& \ddot{\varphi}_{1}=\dot{\varepsilon}_{1} \omega_{\mathrm{m} 0}, \\
& \ddot{\varphi}_{2}=\dot{\varepsilon}_{2} \omega_{\mathrm{m} 0}, \\
& \ddot{\varphi}_{3}=\dot{\varepsilon}_{3} \omega_{\mathrm{m} 0}, \\
& \ddot{\varphi}_{4}=\dot{\varepsilon}_{4} \omega_{\mathrm{m} 0} .
\end{aligned}
$$

where $\gamma_{i}$ is the difference between $\pi$ and the phase angle of the vibrating system in $i$-direction. $\gamma_{i}=$ $\arctan \left(2 \xi_{\mathrm{n} i}\left(\omega_{\mathrm{n} i} / \omega_{\mathrm{m} 0}\right) / 1-\left(\omega_{\mathrm{n} i} / \omega_{\mathrm{m} 0}\right)^{2}\right) . i=x, y, \psi ; m_{0}$ is the mass of the standard exciter; $\eta_{i}$ are the mass ratio of the exciter $i$ to the standard one, $\eta_{i}=m_{i} / m_{0}(i=1,2,3,4)$; $\mu_{i}=1-\left(\omega_{\mathrm{n} i} / \omega_{\mathrm{m} 0}\right)^{2}, i=x, y, \psi ; r_{\mathrm{m}}$ is mass ratio of the standard exciter to the total vibrating system, i.e., $r_{\mathrm{m}}=m_{0} / M$.

Assuming that the derivatives of Equation (4) are $\ddot{x}, \ddot{y}, \dot{\psi}$, and $\ddot{\psi}$, we put them into the last three formulae of Equation (1) and then integrate them with $\varphi=0 \sim 2 \pi$, and the average differential equation of four vibrators is obtained as 


$$
\begin{aligned}
& T_{\mathrm{e} i}-\bar{T}_{\mathrm{L} i}=0, \quad i=1,2,3,4, \\
& \bar{T}_{\mathrm{L} i}=m_{0} r^{2} \omega_{\mathrm{m} 0}\left(\chi_{\mathrm{f} i}+\chi_{\mathrm{a} i}\right), \quad i=1,2,3,4, \\
& \chi_{\mathrm{f} 1}=\frac{\omega_{\mathrm{m} 0}\left[\eta_{1}^{2} W_{\mathrm{s} 0}+\eta_{1} \eta_{2} W_{\mathrm{cs} 12} \cos \left(2 \bar{\alpha}_{1}+\theta_{\mathrm{s} 12}\right)+\eta_{1} \eta_{3} W_{\mathrm{cs} 13} \cos \left(2 \bar{\alpha}_{1}+2 \bar{\alpha}_{2}+\theta_{\mathrm{s} 13}\right)+\eta_{1} \eta_{4} W_{\mathrm{cs} 14} \cos \left(2 \bar{\alpha}_{1}+2 \bar{\alpha}_{2}+2 \bar{\alpha}_{3}+\theta_{\mathrm{s} 14}\right)\right]}{2}, \\
& \chi_{\mathrm{a} 1}=\frac{\omega_{\mathrm{m} 0}\left[\eta_{1} \eta_{2} W_{\mathrm{ccl} 2} \sin \left(2 \alpha_{1}+\theta_{\mathrm{cl} 2}\right)+\eta_{1} \eta_{3} W_{\mathrm{ccl} 13} \sin \left(2 \alpha_{1}+2 \alpha_{2}+\theta_{\mathrm{cl} 3}\right)+\eta_{1} \eta_{4} W_{\mathrm{cc} 14} \sin \left(2 \alpha_{1}+2 \alpha_{2}+2 \alpha_{3}+\theta_{c 14}\right)\right]}{2}, \\
& \chi_{\mathrm{f} 2}=\frac{\omega_{\mathrm{m} 0}\left[\eta_{2}^{2} W_{\mathrm{s} 0}+\eta_{1} \eta_{2} W_{\mathrm{cs} 12} \cos \left(2 \bar{\alpha}_{1}+\theta_{\mathrm{s} 12}\right)+\eta_{2} \eta_{3} W_{\mathrm{cs} 23} \cos \left(2 \bar{\alpha}_{2}+\theta_{\mathrm{s} 23}\right)+\eta_{2} \eta_{4} W_{\mathrm{cs} 24} \cos \left(2 \bar{\alpha}_{2}+2 \bar{\alpha}_{3}+\theta_{\mathrm{s} 24}\right)\right]}{2}, \\
& \chi_{\mathrm{a} 2}=\frac{\omega_{\mathrm{m} 0}\left[-\eta_{1} \eta_{2} W_{\mathrm{cc} 12} \sin \left(2 \alpha_{1}+\theta_{\mathrm{c} 12}\right)+\eta_{2} \eta_{3} W_{\mathrm{cc} 23} \sin \left(2 \alpha_{2}+\theta_{\mathrm{c} 23}\right)+\eta_{2} \eta_{4} W_{\mathrm{cc} 24} \sin \left(2 \alpha_{2}+2 \alpha_{3}+\theta_{\mathrm{c} 24}\right)\right]}{2}, \\
& \chi_{\mathrm{f} 3}=\frac{\omega_{\mathrm{m} 0}\left[\eta_{3}^{2} W_{\mathrm{s} 0}+\eta_{2} \eta_{3} W_{\mathrm{cs} 23} \cos \left(2 \bar{\alpha}_{2}+\theta_{\mathrm{s} 23}\right)+\eta_{1} \eta_{3} W_{\mathrm{cs} 13} \cos \left(2 \bar{\alpha}_{1}+2 \bar{\alpha}_{2}+\theta_{\mathrm{s} 13}\right)+\eta_{3} \eta_{4} W_{\mathrm{cs} 34} \cos \left(2 \bar{\alpha}_{3}+\theta_{\mathrm{s} 34}\right)\right]}{2}, \\
& \chi_{\mathrm{a} 3}=\frac{\omega_{\mathrm{m} 0}\left[-\eta_{2} \eta_{3} W_{\mathrm{cc} 23} \sin \left(2 \alpha_{2}+\theta_{\mathrm{c} 23}\right)-\eta_{1} \eta_{3} W_{\mathrm{cc} 13} \sin \left(2 \alpha_{1}+2 \alpha_{2}+\theta_{\mathrm{c} 13}\right)+\eta_{3} \eta_{4} W_{\mathrm{cc} 34} \sin \left(2 \alpha_{3}+\theta_{\mathrm{c} 34}\right)\right]}{2}, \\
& \chi_{\mathrm{f} 4}=\frac{\omega_{\mathrm{m} 0}\left[\eta_{4}^{2} W_{\mathrm{s} 0}+\eta_{1} \eta_{4} W_{\mathrm{cs} 14} \cos \left(2 \bar{\alpha}_{1}+2 \bar{\alpha}_{2}+2 \bar{\alpha}_{3}+\theta_{\mathrm{s} 14}\right)+\eta_{2} \eta_{4} W_{\mathrm{cs} 24} \cos \left(2 \bar{\alpha}_{2}+2 \bar{\alpha}_{3}+\theta_{\mathrm{s} 24}\right)+\eta_{3} \eta_{4} W_{\mathrm{cs} 34} \cos \left(2 \bar{\alpha}_{3}+\theta_{\mathrm{s} 34}\right)\right]}{2}, \\
& \chi_{\mathrm{a} 4}=\frac{\omega_{\mathrm{m} 0}\left[-\eta_{1} \eta_{4} W_{\mathrm{cc} 14} \sin \left(2 \alpha_{1}+2 \alpha_{2}+2 \alpha_{3}+\theta_{\mathrm{c} 14}\right)-\eta_{2} \eta_{4} W_{\mathrm{cc} 24} \sin \left(2 \alpha_{2}+2 \alpha_{3}+\theta_{\mathrm{c} 24}\right)-\eta_{3} \eta_{4} W_{\mathrm{cc} 34} \sin \left(2 \alpha_{3}+\theta_{\mathrm{c} 34}\right)\right]}{2}, \\
& W_{\mathrm{s} 0}=r_{\mathrm{m}}\left(\frac{r_{10}^{2} \sin \left(\gamma_{\psi}\right)}{\mu_{\psi}}+\frac{\sin \left(\gamma_{x}\right)}{\mu_{x}}+\frac{\sin \left(\gamma_{y}\right)}{\mu_{y}}\right) \\
& W_{\mathrm{c} 0}=r_{\mathrm{m}}\left(\frac{r_{10}^{2} \cos \left(\gamma_{\psi}\right)}{\mu_{\psi}}+\frac{\cos \left(\gamma_{x}\right)}{\mu_{x}}+\frac{\cos \left(\gamma_{y}\right)}{\mu_{y}}\right) \text {, } \\
& a_{\mathrm{csij}}=\frac{\sin \gamma_{x}}{\mu_{x}}+\frac{\sin \gamma_{y}}{\mu_{y}}+\frac{r_{1}^{2} \sin \gamma_{\psi}}{\mu_{\psi}} \cos \left(\beta_{i}-\beta_{j}\right), \quad i=1,2,3 ; \quad i<j \leq 4, \\
& b_{\mathrm{csij}}=\frac{r_{1}^{2} \sin \gamma_{\psi}}{\mu_{\psi}} \sin \left(\beta_{i}-\beta_{j}\right), \quad i=1,2,3 ; \quad i<j \leq 4, \\
& a_{\mathrm{ccij}}=-\frac{\cos \gamma_{x}}{\mu_{x}}-\frac{\cos \gamma_{y}}{\mu_{y}}-\frac{r_{1}^{2} \cos \gamma_{\psi}}{\mu_{\psi}} \cos \left(\beta_{i}-\beta_{j}\right), \quad i=1,2,3 ; \quad i<j \leq 4, \\
& b_{\mathrm{ccij}}=\frac{r_{1}^{2} \cos \gamma_{\psi}}{\mu_{\psi}} \sin \left(\beta_{i}-\beta_{j}\right), \quad i=1,2,3 ; \quad i<j \leq 4, \\
& W_{\mathrm{csij}}=r_{\mathrm{m}} \sqrt{a_{\mathrm{cs} i j}^{2}+b_{\mathrm{cs} i j}^{2}}, \quad i=1,2,3 ; \quad i<j \leq 4, \\
& W_{\mathrm{ccij}}=r_{\mathrm{m}} \sqrt{a_{\mathrm{ccij}}^{2}+b_{\mathrm{ccij}}^{2}}, \quad i=1,2,3 ; \quad i<j \leq 4, \\
& \theta_{\mathrm{s} i j}=\left\{\begin{array}{l}
\arctan \left(-b_{\mathrm{csij}} / a_{\mathrm{csij}}\right), \quad a_{\mathrm{cs} i j} \geq 0 ; \\
\pi+\arctan \left(-b_{\mathrm{csij}} / a_{\mathrm{cs} i j}\right), \quad a_{\mathrm{cs} i j}<0 ;
\end{array} \quad i=1,2,3 ; \quad i<j \leq 4,\right. \\
& \theta_{c i j}=\left\{\begin{array}{l}
\arctan \left(b_{\mathrm{ccij}} / a_{\mathrm{ccij}}\right), \quad a_{\mathrm{ccij}} \geq 0 ; \\
\pi+\arctan \left(b_{\mathrm{ccij}} / a_{\mathrm{cc} i j}\right), \quad a_{\mathrm{cc} i j}<0 ;
\end{array} \quad i=1,2,3 ; \quad i<j \leq 4 .\right.
\end{aligned}
$$


3.1. Synchronization Criterion. Based on the formula of electromagnetic torque, the frequency capture equation is obtained. When four vibrators can operate synchronously, we can obtain the output electromagnetic torque $T_{0 i}$ as follows:

$$
T_{0 i}=T_{\mathrm{e} 0 i}-f_{i} \omega_{\mathrm{m} 0}=m_{0} r^{2} \omega_{\mathrm{m} 0}\left(\chi_{\mathrm{f} i}+\chi_{\mathrm{a} i}\right), \quad i=1,2,3,4,
$$

where $T_{0 i}$ indicates the differences between the electromagnetic torque of the motor $i$ and the damping torque of its rotor.

From Equation (6), the differences of output torque between the motors 1 and $2\left(\Delta T_{012}\right), 2$ and $3\left(\Delta T_{023}\right)$, and 3 and $4\left(\Delta T_{034}\right)$ can be expressed as

$$
\begin{aligned}
& \Delta T_{012}=T_{01}-T_{02}=T_{\mathrm{u}}\left[\left(\eta_{1}^{2}-\eta_{2}^{2}\right) W_{\mathrm{s} 0}\right. \\
& +\eta_{1} \eta_{3} W_{\mathrm{cs} 13} \cos \left(2 \bar{\alpha}_{1}+2 \bar{\alpha}_{2}+\theta_{\mathrm{s} 13}\right) \\
& -\eta_{2} \eta_{3} W_{\mathrm{cs} 23} \cos \left(2 \bar{\alpha}_{2}+\theta_{\mathrm{s} 23}\right) \\
& +\eta_{1} \eta_{4} W_{\mathrm{cs} 14} \cos \left(2 \bar{\alpha}_{1}+2 \bar{\alpha}_{2}+2 \bar{\alpha}_{3}+\theta_{\mathrm{s} 14}\right) \\
& -\eta_{2} \eta_{4} W_{\mathrm{cs} 24} \cos \left(2 \bar{\alpha}_{2}+2 \bar{\alpha}_{3}+\theta_{\mathrm{s} 24}\right) \\
& +2 \eta_{1} \eta_{2} W_{\mathrm{cc} 12} \sin \left(2 \bar{\alpha}_{1}+\theta_{\mathrm{c} 12}\right) \\
& +\eta_{1} \eta_{3} W_{\mathrm{cc} 13} \sin \left(2 \bar{\alpha}_{1}+2 \bar{\alpha}_{2}+\theta_{\mathrm{cl} 3}\right) \\
& -\eta_{2} \eta_{3} W_{\mathrm{cc} 23} \sin \left(2 \bar{\alpha}_{2}+\theta_{\mathrm{c} 23}\right) \\
& +\eta_{1} \eta_{4} W_{\mathrm{cc} 14} \sin \left(2 \bar{\alpha}_{1}+2 \bar{\alpha}_{2}+2 \bar{\alpha}_{3}+\theta_{\mathrm{cl} 14}\right) \\
& \left.-\eta_{2} \eta_{4} W_{\mathrm{cc} 24} \sin \left(2 \bar{\alpha}_{2}+2 \bar{\alpha}_{3}+\theta_{\mathrm{c} 24}\right)\right] \text {, } \\
& \Delta T_{023}=T_{02}-T_{03}=T_{\mathrm{u}}\left[\eta_{2}^{2}-\eta_{3}^{2}\right) W_{\mathrm{s} 0} \\
& +\eta_{1} \eta_{2} W_{\mathrm{cs} 12} \cos \left(2 \bar{\alpha}_{1}+\theta_{\mathrm{s} 12}\right) \\
& -\eta_{1} \eta_{3} W_{\mathrm{cs} 13} \cos \left(2 \bar{\alpha}_{1}+2 \bar{\alpha}_{2}+\theta_{\mathrm{s} 13}\right) \\
& +\eta_{2} \eta_{4} W_{\mathrm{cs} 24} \cos \left(2 \bar{\alpha}_{2}+2 \bar{\alpha}_{3}+\theta_{\mathrm{s} 24}\right) \\
& -\eta_{3} \eta_{4} W_{\mathrm{cs} 34} \cos \left(2 \bar{\alpha}_{3}+\theta_{\mathrm{s} 34}\right) \\
& +2 \eta_{2} \eta_{3} W_{\mathrm{cc} 23} \sin \left(2 \bar{\alpha}_{2}+\theta_{\mathrm{c} 23}\right) \\
& +\eta_{1} \eta_{3} W_{\mathrm{cc} 13} \sin \left(2 \bar{\alpha}_{1}+2 \bar{\alpha}_{2}+\theta_{\mathrm{cl} 3}\right) \\
& -\eta_{1} \eta_{2} W_{\mathrm{cc} 12} \sin \left(2 \bar{\alpha}_{1}+\theta_{\mathrm{cc} 12}\right) \\
& +\eta_{2} \eta_{4} W_{\mathrm{cc} 24} \sin \left(2 \bar{\alpha}_{2}+2 \bar{\alpha}_{3}+\theta_{\mathrm{c} 24}\right) \\
& \left.-\eta_{3} \eta_{4} W_{\mathrm{cc} 34} \sin \left(2 \bar{\alpha}_{3}+\theta_{\mathrm{c} 34}\right)\right] \text {, } \\
& \Delta T_{034}=T_{03}-T_{04}=T_{\mathrm{u}}\left[\left(\eta_{3}^{2}-\eta_{4}^{2}\right) W_{\mathrm{s} 0}\right. \\
& +\eta_{2} \eta_{3} W_{\mathrm{cs} 23} \cos \left(2 \bar{\alpha}_{2}+\theta_{\mathrm{s} 23}\right) \\
& -\eta_{2} \eta_{4} W_{\mathrm{cs} 24} \cos \left(2 \bar{\alpha}_{2}+2 \bar{\alpha}_{3}+\theta_{\mathrm{s} 24}\right) \\
& +\eta_{1} \eta_{3} W_{\mathrm{cs} 13} \cos \left(2 \bar{\alpha}_{1}+2 \bar{\alpha}_{2}+\theta_{\mathrm{s} 13}\right) \\
& -\eta_{1} \eta_{4} W_{\mathrm{cs} 14} \cos \left(2 \bar{\alpha}_{1}+2 \bar{\alpha}_{2}+2 \bar{\alpha}_{3}+\theta_{\mathrm{s} 14}\right) \\
& -\eta_{2} \eta_{3} W_{\mathrm{cc} 23} \sin \left(2 \bar{\alpha}_{2}+\theta_{\mathrm{c} 23}\right) \\
& -\eta_{1} \eta_{3} W_{\mathrm{cc} 13} \sin \left(2 \bar{\alpha}_{1}+2 \bar{\alpha}_{2}+\theta_{\mathrm{cl} 3}\right) \\
& +2 \eta_{3} \eta_{4} W_{\mathrm{cc} 34} \sin \left(2 \bar{\alpha}_{3}+\theta_{\mathrm{c} 34}\right) \\
& +\eta_{2} \eta_{4} W_{\mathrm{cc} 24} \sin \left(2 \bar{\alpha}_{2}+2 \bar{\alpha}_{3}+\theta_{\mathrm{c} 24}\right) \\
& \left.+\eta_{1} \eta_{4} W_{\mathrm{cc} 14} \sin \left(2 \bar{\alpha}_{1}+2 \bar{\alpha}_{2}+2 \bar{\alpha}_{3}+\theta_{\mathrm{c} 14}\right)\right]
\end{aligned}
$$

where $T_{\mathrm{su}}=m_{0} r^{2} \omega_{\mathrm{m} 0}^{2} / 2$ indicates the kinetic energy of the standard vibrator.

Through rearrangement, Equation (7) is expressed as

$$
\begin{aligned}
& \frac{\Delta T_{012}}{T_{\mathrm{SU}}}-\left(\eta_{1}^{2}-\eta_{2}^{2}\right) W_{\mathrm{S} 0}=\tau_{\mathrm{c} 12}\left(\bar{\alpha}_{1}, \bar{\alpha}_{2}, \bar{\alpha}_{3}\right), \\
& \frac{\Delta T_{023}}{T_{\mathrm{SU}}}-\left(\eta_{2}^{2}-\eta_{3}^{2}\right) W_{\mathrm{S} 0}=\tau_{\mathrm{c} 23}\left(\bar{\alpha}_{1}, \bar{\alpha}_{2}, \bar{\alpha}_{3}\right), \\
& \frac{\Delta T_{034}}{T_{\mathrm{SU}}}-\left(\eta_{3}^{2}-\eta_{4}^{2}\right) W_{\mathrm{S} 0}=\tau_{\mathrm{c} 34}\left(\bar{\alpha}_{1}, \bar{\alpha}_{2}, \bar{\alpha}_{3}\right) .
\end{aligned}
$$

The coefficients $\tau_{c 12}\left(\bar{\alpha}_{1}, \bar{\alpha}_{2}, \bar{\alpha}_{3}\right), \tau_{\mathrm{c} 23}\left(\bar{\alpha}_{1}, \bar{\alpha}_{2}, \bar{\alpha}_{3}\right)$, $\tau_{\mathrm{c} 34}\left(\bar{\alpha}_{1}, \bar{\alpha}_{2}, \bar{\alpha}_{3}\right)$ of Equations (8)-(10) are given by

$$
\begin{aligned}
& \tau_{\mathrm{c} 12}\left(\bar{\alpha}_{1}, \bar{\alpha}_{2}, \bar{\alpha}_{3}\right)=\eta_{1} \eta_{3} W_{\mathrm{cs} 13} \cos \left(2 \bar{\alpha}_{1}+2 \bar{\alpha}_{2}+\theta_{s 13}\right) \\
& -\eta_{2} \eta_{3} W_{\mathrm{cs} 23} \cos \left(2 \bar{\alpha}_{2}+\theta_{\mathrm{s} 13}\right) \\
& +\eta_{1} \eta_{4} W_{\mathrm{cs} 14} \cos \left(2 \bar{\alpha}_{1}+2 \bar{\alpha}_{2}+2 \bar{\alpha}_{3}+\theta_{\mathrm{s} 14}\right) \\
& -\eta_{2} \eta_{4} W_{\mathrm{cs} 24} \cos \left(2 \bar{\alpha}_{2}+2 \bar{\alpha}_{3}+\theta_{\mathrm{s} 24}\right) \\
& +2 \eta_{1} \eta_{2} W_{\mathrm{cc} 12} \sin \left(2 \bar{\alpha}_{1}+\theta_{\mathrm{c} 12}\right) \\
& +\eta_{1} \eta_{3} W_{\mathrm{cc} 13} \sin \left(2 \bar{\alpha}_{1}+2 \bar{\alpha}_{2}+\theta_{\mathrm{c} 13}\right) \\
& -\eta_{2} \eta_{3} W_{\mathrm{cc} 23} \sin \left(2 \bar{\alpha}_{2}+\theta_{\mathrm{c} 23}\right) \\
& +\eta_{1} \eta_{4} W_{\mathrm{cc} 14} \sin \left(2 \bar{\alpha}_{1}+2 \bar{\alpha}_{2}+2 \bar{\alpha}_{3}+\theta_{\mathrm{c} 14}\right) \\
& -\eta_{2} \eta_{4} W_{\mathrm{cc} 24} \sin \left(2 \bar{\alpha}_{2}+2 \bar{\alpha}_{3}+\theta_{\mathrm{c} 24}\right) \text {, } \\
& \tau_{\mathrm{c} 23}\left(\bar{\alpha}_{1}, \bar{\alpha}_{2}, \bar{\alpha}_{3}\right)=\eta_{1} \eta_{2} W_{\mathrm{cs} 12} \cos \left(2 \bar{\alpha}_{1}+\theta_{\mathrm{s} 12}\right) \\
& -\eta_{1} \eta_{3} W_{\mathrm{cs} 13} \cos \left(2 \bar{\alpha}_{1}+2 \bar{\alpha}_{2}+\theta_{\mathrm{s} 13}\right) \\
& +\eta_{2} \eta_{4} W_{\mathrm{cs} 24} \cos \left(2 \bar{\alpha}_{2}+2 \bar{\alpha}_{3}+\theta_{\mathrm{s} 24}\right) \\
& -\eta_{3} \eta_{4} W_{\mathrm{cs} 34} \cos \left(2 \bar{\alpha}_{3}+\theta_{\mathrm{s} 34}\right) \\
& +2 \eta_{2} \eta_{3} W_{\mathrm{cc} 23} \sin \left(2 \bar{\alpha}_{2}+\theta_{\mathrm{c} 23}\right) \\
& +\eta_{1} \eta_{3} W_{\mathrm{cc} 13} \sin \left(2 \bar{\alpha}_{1}+2 \bar{\alpha}_{2}+\theta_{\mathrm{c} 13}\right) \\
& -\eta_{1} \eta_{2} W_{\mathrm{cc} 12} \sin \left(2 \bar{\alpha}_{1}+\theta_{\mathrm{c} 12}\right) \\
& +\eta_{2} \eta_{4} W_{\mathrm{cc} 24} \sin \left(2 \bar{\alpha}_{2}+2 \bar{\alpha}_{3}+\theta_{\mathrm{c} 24}\right) \\
& -\eta_{3} \eta_{4} W_{\mathrm{cc} 34} \sin \left(2 \bar{\alpha}_{3}+\theta_{\mathrm{c} 24}\right) \\
& \tau_{\mathrm{c} 34}\left(\bar{\alpha}_{1}, \bar{\alpha}_{2}, \bar{\alpha}_{3}\right)=\eta_{2} \eta_{3} W_{\mathrm{cs} 23} \cos \left(2 \bar{\alpha}_{2}+\theta_{\mathrm{s} 23}\right) \\
& -\eta_{2} \eta_{4} W_{\mathrm{cs} 24} \cos \left(2 \bar{\alpha}_{2}+2 \bar{\alpha}_{3}+\theta_{\mathrm{s} 24}\right) \\
& +\eta_{1} \eta_{3} W_{\mathrm{cs} 13} \cos \left(2 \bar{\alpha}_{1}+2 \bar{\alpha}_{2}+\theta_{\mathrm{s} 13}\right) \\
& -\eta_{1} \eta_{4} W_{\mathrm{cs} 14} \cos \left(2 \bar{\alpha}_{1}+2 \bar{\alpha}_{2}+2 \bar{\alpha}_{3}+\theta_{\mathrm{s} 14}\right) \\
& -\eta_{2} \eta_{3} W_{\mathrm{cc} 23} \sin \left(2 \bar{\alpha}_{2}+\theta_{\mathrm{c} 23}\right) \\
& -\eta_{1} \eta_{3} W_{\mathrm{cc} 13} \sin \left(2 \bar{\alpha}_{1}+2 \bar{\alpha}_{2}+\theta_{\mathrm{cl} 3}\right) \\
& +2 \eta_{3} \eta_{4} W_{\mathrm{cc} 34} \sin \left(2 \bar{\alpha}_{3}+\theta_{\mathrm{c} 34}\right) \\
& +\eta_{2} \eta_{4} W_{\mathrm{cc} 24} \sin \left(2 \bar{\alpha}_{2}+2 \bar{\alpha}_{3}+\theta_{\mathrm{c} 24}\right) \\
& +\eta_{1} \eta_{4} W_{\mathrm{cc} 14} \sin \left(2 \bar{\alpha}_{1}+2 \bar{\alpha}_{2}+2 \bar{\alpha}_{3}+\theta_{\mathrm{c} 14}\right) \text {. }
\end{aligned}
$$

$\tau_{\text {cij }}\left(\bar{\alpha}_{1}, \bar{\alpha}_{2}, \bar{\alpha}_{3}\right)(i j=12,23,34)$ denote the dimensionless coupling torque between vibrators $i$ and $j$, which are limited functions of $\bar{\alpha}_{1}, \bar{\alpha}_{2}$, and $\bar{\alpha}_{3}$, that is, 


$$
\begin{aligned}
& \left|\tau_{\mathrm{c} 12}\left(\bar{\alpha}_{1}, \bar{\alpha}_{2}, \bar{\alpha}_{3}\right)\right| \leq \tau_{\mathrm{c} 12 \max }, \\
& \left|\tau_{\mathrm{c} 23}\left(\bar{\alpha}_{1}, \bar{\alpha}_{2}, \bar{\alpha}_{3}\right)\right| \leq \tau_{\mathrm{c} 23 \max }, \\
& \left|\tau_{\mathrm{c} 34}\left(\bar{\alpha}_{1}, \bar{\alpha}_{2}, \bar{\alpha}_{3}\right)\right| \leq \tau_{\mathrm{c} 34 \max }
\end{aligned}
$$
obtain

Considering Equations (8)-(10) and (12)-(14), we can

$$
\begin{aligned}
& \left|\frac{\Delta T_{012}}{T_{\mathrm{su}}}-\left(\eta_{1}^{2}-\eta_{2}^{2}\right) W_{\mathrm{s} 0}\right| \leq \tau_{\mathrm{c} 12 \max }, \\
& \left|\frac{\Delta T_{023}}{T_{\mathrm{su}}}-\left(\eta_{2}^{2}-\eta_{3}^{2}\right) W_{\mathrm{s} 0}\right| \leq \tau_{\mathrm{c} 23 \max }, \\
& \left|\frac{\Delta T_{034}}{T_{\mathrm{su}}}-\left(\eta_{3}^{2}-\eta_{4}^{2}\right) W_{\mathrm{s} 0}\right| \leq \tau_{\mathrm{c} 34 \max } .
\end{aligned}
$$

Equations (15)-(17) denote the synchronization criterion of four vibrators. The left sides of inequalities show the absolute value of dimensionless residual torque differences between arbitrary two motors, and their right sides represent the maximum of dimensionless coupling torque.

The sum of Equation (6) leads to

$$
\begin{aligned}
\tau_{\mathrm{a}}\left(\bar{\alpha}_{1}, \bar{\alpha}_{2}, \bar{\alpha}_{3}\right)= & \frac{1}{4 T_{\mathrm{su}}} \sum_{i=1}^{4} T_{\mathrm{o} i}=\frac{1}{4}\left[\sum_{i=1}^{4} \eta_{i}^{2} W_{\mathrm{s} 0}\right. \\
& +2 \eta_{1} \eta_{2} W_{\mathrm{cs} 12} \cos \left(2 \bar{\alpha}_{1}+\theta_{\mathrm{s} 12}\right) \\
& +2 \eta_{2} \eta_{3} W_{\mathrm{cs} 23} \cos \left(2 \bar{\alpha}_{2}+\theta_{\mathrm{s} 23}\right) \\
& +2 \eta_{3} \eta_{4} W_{\mathrm{cs} 34} \cos \left(2 \bar{\alpha}_{3}+\theta_{\mathrm{s} 34}\right) \\
& +2 \eta_{1} \eta_{4} W_{\mathrm{cs} 14} \cos \left(2 \bar{\alpha}_{1}+2 \bar{\alpha}_{2}+2 \bar{\alpha}_{3}+\theta_{\mathrm{s} 14}\right) \\
& +2 \eta_{1} \eta_{3} W_{\mathrm{cs} 13} \cos \left(2 \bar{\alpha}_{1}+2 \bar{\alpha}_{2}+\theta_{\mathrm{s} 13}\right) \\
& \left.+2 \eta_{2} \eta_{4} W_{\mathrm{cs} 24} \cos \left(2 \bar{\alpha}_{2}+2 \bar{\alpha}_{3}+\theta_{\mathrm{s} 24}\right)\right]
\end{aligned}
$$

where $\tau_{\mathrm{a}}\left(\bar{\alpha}_{1}, \bar{\alpha}_{2}, \bar{\alpha}_{3}\right)$ indicates the average dimensionless loading torque of four motors. As a limited function, it satisfies the following formula:

$$
\left|\tau_{\mathrm{a}}\left(\bar{\alpha}_{1}, \bar{\alpha}_{2}, \bar{\alpha}_{3}\right)\right| \leq \tau_{\mathrm{a} \max },
$$

where $\tau_{\text {a max }}$ denotes the maximum of the average dimensionless loading torque of four motors.

We define that $\zeta_{i j}$ denotes the coefficient of synchronization ability, that is,

$$
\begin{aligned}
& \zeta_{12}=\frac{\tau_{\mathrm{c} 12 \max }}{\tau_{\mathrm{a} \max }} \\
& \zeta_{23}=\frac{\tau_{\mathrm{c} 23 \max }}{\tau_{\mathrm{a} \max }} \\
& \zeta_{34}=\frac{\tau_{\mathrm{c} 34 \max }}{\tau_{\mathrm{a} \max }}, \\
& \zeta_{41}=\frac{\tau_{\mathrm{c} 41 \max }}{\tau_{\mathrm{a} \max }},
\end{aligned}
$$

with

$$
\begin{aligned}
\left|\tau_{\mathrm{c} 41}\left(\bar{\alpha}_{1}, \bar{\alpha}_{2}, \bar{\alpha}_{3}\right)\right| \leq & \tau_{\mathrm{c} 41 \mathrm{max}}, \\
\tau_{\mathrm{c} 41}\left(\bar{\alpha}_{1}, \bar{\alpha}_{2}, \bar{\alpha}_{3}\right)= & -\left[\tau_{\mathrm{c} 12}\left(\bar{\alpha}_{1}, \bar{\alpha}_{2}, \bar{\alpha}_{3}\right)+\tau_{\mathrm{c} 23}\left(\bar{\alpha}_{1}, \bar{\alpha}_{2}, \bar{\alpha}_{3}\right)\right. \\
& \left.+\tau_{\mathrm{c} 34}\left(\bar{\alpha}_{1}, \bar{\alpha}_{2}, \bar{\alpha}_{3}\right)\right] .
\end{aligned}
$$

The larger the above coefficients, the stronger the coupling torques among vibrators. In other words, the larger these coefficients $\zeta_{12}, \zeta_{23}, \zeta_{34}$, and $\zeta_{41}$, the more easily vibrators can achieve synchronization.

$\tau_{c i j}\left(\bar{\alpha}_{1}, \bar{\alpha}_{2}, \bar{\alpha}_{3}\right)(i j=12,23,34)$ and $\tau_{\mathrm{a}}\left(\bar{\alpha}_{1}, \bar{\alpha}_{2}, \bar{\alpha}_{3}\right)$ are decided by parameters of the system, and their maximum will be discussed numerically in Section 4 .

3.2. Stability Criterion of the Synchronous States. According to Equation (3), the following formulae are obtained:

$$
\begin{aligned}
& v_{1}=\frac{3}{2} \alpha_{1}+\alpha_{2}+\frac{1}{2} \alpha_{3}, \\
& v_{2}=-\frac{1}{2} \alpha_{1}+\alpha_{2}+\frac{1}{2} \alpha_{3}, \\
& v_{3}=-\frac{1}{2} \alpha_{1}-\alpha_{2}+\frac{1}{2} \alpha_{3}, \\
& v_{4}=-\frac{1}{2} \alpha_{1}-\alpha_{2}-\frac{3}{2} \alpha_{3} .
\end{aligned}
$$

Assuming $\Delta v_{i}=v_{i}-v_{i 0}$ and linearizing $\left(\Delta v_{i}\right)^{\prime}$ around $\bar{\alpha}_{10}, \bar{\alpha}_{20}, \bar{\alpha}_{30}$, we can obtain

$$
\begin{aligned}
& \left(\Delta v_{1}\right)^{\prime}=-\sum_{i=1}^{3}\left(\frac{\partial \chi_{\mathrm{a} 1}}{\partial \alpha_{i}}\right)_{0}\left(\Delta \alpha_{i}\right), \\
& \left(\Delta v_{2}\right)^{\prime}=-\sum_{i=1}^{3}\left(\frac{\partial \chi_{a 2}}{\partial \alpha_{i}}\right)_{0}\left(\Delta \alpha_{i}\right), \\
& \left(\Delta v_{3}\right)^{\prime}=-\sum_{i=1}^{3}\left(\frac{\partial \chi_{a 3}}{\partial \alpha_{i}}\right)_{0}\left(\Delta \alpha_{i}\right), \\
& \left(\Delta v_{4}\right)^{\prime}=-\sum_{i=1}^{3}\left(\frac{\partial \chi_{a 4}}{\partial \alpha_{i}}\right)_{0}\left(\Delta \alpha_{i}\right),
\end{aligned}
$$

where $(\cdot)_{0}$ denotes the values for $\bar{\alpha}_{1}=\bar{\alpha}_{10}, \bar{\alpha}_{2}=\bar{\alpha}_{20}, \bar{\alpha}_{3}=$ $\bar{\alpha}_{30}$, and $\Delta \alpha_{i}=\bar{\alpha}_{i}-\bar{\alpha}_{i 0}, \quad i=1,2,3$.

Through rearrangement, Equation (23) is expressed as

$$
\Delta \dot{\alpha}=\mathbf{D} \Delta \boldsymbol{\alpha},
$$


with

$$
\begin{aligned}
& \mathbf{D}=\left(d_{i j}\right)_{3 \times 3} \\
& d_{11}=\left(-\frac{\omega_{\mathrm{m} 0}^{*}}{2}\right)\left[\frac{\eta_{1} \eta_{2}\left(\left(1 / k_{11}\right)+\left(1 / k_{22}\right)\right) W_{\mathrm{cc} 12} \cos \left(2 \bar{\alpha}_{10}+\theta_{\mathrm{cl} 2}\right)+\eta_{1} \eta_{3} W_{\mathrm{cc} 13} \cos \left(2 \bar{\alpha}_{10}+2 \bar{\alpha}_{20}+\theta_{\mathrm{cl} 3}\right)}{k_{11}}\right. \\
& \left.+\frac{\eta_{1} \eta_{4} W_{\mathrm{cc} 14} \cos \left(2 \bar{\alpha}_{10}+2 \bar{\alpha}_{20}+2 \bar{\alpha}_{30}+\theta_{\mathrm{c} 14}\right)}{k_{11}}\right] \\
& d_{12}=\left(\frac{\omega_{\mathrm{m} 0}^{*}}{2}\right)\left[\frac{\eta_{2} \eta_{3} W_{\mathrm{cc} 23} \cos \left(2 \bar{\alpha}_{20}+\theta_{\mathrm{c} 23}\right)}{k_{22}}+\frac{\eta_{2} \eta_{4} W_{\mathrm{cc} 24} \cos \left(2 \bar{\alpha}_{20}+2 \bar{\alpha}_{30}+\theta_{\mathrm{c} 24}\right)}{k_{22}}-\frac{\eta_{1} \eta_{3} W_{\mathrm{cc} 13} \cos \left(2 \bar{\alpha}_{10}+2 \bar{\alpha}_{20}+\theta_{\mathrm{cl3}}\right)}{k_{11}}\right. \\
& \left.-\frac{\eta_{1} \eta_{4} W_{\mathrm{cc} 14} \cos \left(2 \bar{\alpha}_{10}+2 \bar{\alpha}_{20}+2 \bar{\alpha}_{30}+\theta_{\mathrm{c} 14}\right)}{k_{11}}\right] \\
& d_{13}=\left(\frac{\omega_{\mathrm{m} 0}^{*}}{2}\right)\left[\frac{\eta_{2} \eta_{4} W_{\mathrm{cc} 24} \cos \left(2 \bar{\alpha}_{20}+2 \bar{\alpha}_{30}+\theta_{\mathrm{c} 24}\right)}{k_{22}}-\frac{\eta_{1} \eta_{4} W_{\mathrm{cc} 14} \cos \left(2 \bar{\alpha}_{10}+2 \bar{\alpha}_{20}+2 \bar{\alpha}_{30}+\theta_{\mathrm{c} 14}\right)}{k_{11}}\right], \\
& d_{21}=\left(\frac{\omega_{\mathrm{m} 0}^{*}}{2}\right)\left[\frac{\eta_{1} \eta_{2} W_{c c 12} \cos \left(2 \bar{\alpha}_{10}+\theta_{c 12}\right)}{k_{22}}-\frac{\eta_{1} \eta_{3} W_{c c 13} \cos \left(2 \bar{\alpha}_{10}+2 \bar{\alpha}_{20}+\theta_{c 13}\right)}{k_{33}}\right] \text {, } \\
& d_{22}=\left(-\frac{\omega_{\mathrm{m} 0}^{*}}{2}\right)\left[\frac{\eta_{2} \eta_{3}\left(\left(1 / k_{22}\right)+\left(1 / k_{33}\right)\right) W_{\mathrm{cc} 23} \cos \left(2 \bar{\alpha}_{20}+\theta_{\mathrm{c} 23}\right)+\eta_{2} \eta_{4} W_{\mathrm{cc} 24} \cos \left(2 \bar{\alpha}_{20}+2 \bar{\alpha}_{30}+\theta_{\mathrm{c} 24}\right)}{k_{22}}\right. \\
& \left.+\frac{\eta_{1} \eta_{3} W_{\mathrm{cc} 13} \cos \left(2 \bar{\alpha}_{10}+2 \bar{\alpha}_{20}+\theta_{\mathrm{cl} 13}\right)}{k_{33}}\right] \\
& d_{31}=\left(\frac{\omega_{\mathrm{m} 0}^{*}}{2}\right)\left[\frac{\eta_{1} \eta_{3} W_{\mathrm{cc} 13} \cos \left(2 \bar{\alpha}_{10}+2 \bar{\alpha}_{20}+\theta_{\mathrm{cl} 3}\right)}{k_{33}}-\frac{\eta_{1} \eta_{4} W_{\mathrm{cc} 14} \cos \left(2 \bar{\alpha}_{10}+2 \bar{\alpha}_{20}+2 \bar{\alpha}_{30}+\theta_{\mathrm{cl} 14}\right)}{k_{44}}\right], \\
& d_{32}=\left(\frac{\omega_{\mathrm{m} 0}^{*}}{2}\right)\left[\frac{\eta_{2} \eta_{3} W_{\mathrm{cc} 23} \cos \left(2 \bar{\alpha}_{20}+\theta_{\mathrm{c} 23}\right)}{k_{33}}+\frac{\eta_{1} \eta_{3} W_{\mathrm{cc} 13} \cos \left(2 \bar{\alpha}_{10}+2 \bar{\alpha}_{20}+\theta_{\mathrm{cl} 13}\right)}{k_{33}}-\frac{\eta_{1} \eta_{4} W_{\mathrm{cc} 14} \cos \left(2 \bar{\alpha}_{10}+2 \bar{\alpha}_{20}+2 \bar{\alpha}_{30}+\theta_{\mathrm{cl} 14}\right)}{k_{44}}\right. \\
& \left.-\frac{\eta_{2} \eta_{4} W_{\mathrm{cc} 24} \cos \left(2 \bar{\alpha}_{20}+2 \bar{\alpha}_{30}+\theta_{\mathrm{c} 24}\right)}{k_{44}}\right] \text {, } \\
& d_{33}=\left(-\frac{\omega_{\mathrm{m} 0}^{*}}{2}\right)\left[\frac{\eta_{3} \eta_{4}\left(\left(1 / k_{33}\right)+\left(1 / k_{44}\right)\right) W_{\mathrm{cc} 34} \cos \left(2 \bar{\alpha}_{30}+\theta_{\mathrm{c} 23}\right)+\eta_{1} \eta_{4} W_{\mathrm{cc} 14} \cos \left(2 \bar{\alpha}_{10}+2 \bar{\alpha}_{20}+2 \bar{\alpha}_{30}+\theta_{\mathrm{c} 14}\right)}{k_{44}}\right. \\
& \left.+\frac{\eta_{2} \eta_{4} W_{c c 24} \cos \left(2 \bar{\alpha}_{20}+2 \bar{\alpha}_{30}+\theta_{c 24}\right)}{k_{44}}\right] \text {. }
\end{aligned}
$$

We assume $\Delta \boldsymbol{\alpha}=\mathbf{v} \exp (\lambda t)$ and insert it into Equation (24) and then solve the equation $\operatorname{det}(\mathbf{D}-\lambda \mathbf{I})$; the characteristic equation for eigenvalue $\lambda$ is obtained as follows:

$$
\begin{aligned}
\lambda^{3}- & \left(d_{11}+d_{22}+d_{33}\right) \lambda^{2}+\left(d_{11} d_{33}+d_{22} d_{33}+d_{11} d_{22}\right. \\
& \left.-d_{13} d_{31}-d_{23} d_{32}-d_{12} d_{21}\right) \lambda-\left(d_{11} d_{22} d_{33}+d_{12} d_{23} d_{31}\right. \\
& \left.+d_{13} d_{21} d_{32}-d_{13} d_{22} d_{31}-d_{11} d_{23} d_{32}-d_{12} d_{21} d_{33}\right)=0 .
\end{aligned}
$$

Considering to introduce additional parameters

$$
\begin{aligned}
a= & -d_{11}-d_{22}-d_{33}, \\
b= & d_{11} d_{33}+d_{22} d_{33}+d_{11} d_{22}-d_{13} d_{31}-d_{23} d_{32}-d_{12} d_{21}, \\
e= & -\left(d_{11} d_{22} d_{33}+d_{12} d_{23} d_{31}+d_{13} d_{21} d_{32}-d_{13} d_{22} d_{31}\right. \\
& \left.-d_{11} d_{23} d_{32}-d_{12} d_{21} d_{33}\right) .
\end{aligned}
$$


After the rearrangement, we can deduce

$$
\lambda^{3}+a \lambda^{2}+b \lambda+e=0 \text {. }
$$

Applying the Routh-Hurwitz criterion, we deduce the criterion of stability of the synchronous states in the following equation:

$$
\begin{aligned}
a & >0, \\
a b-e & >0, \\
e & >0 .
\end{aligned}
$$

Substituting the parameters $a, b$, and $e$ into Equation (29) yields

$$
\begin{aligned}
H_{1}= & -d_{11}-d_{22}-d_{33}>0, \\
H_{2}= & d_{11} d_{33}+d_{22} d_{33}+d_{11} d_{22}-d_{13} d_{31}-d_{23} d_{32}-d_{12} d_{21}>0, \\
H_{3}= & -\left(d_{11} d_{22} d_{33}+d_{12} d_{23} d_{31}+d_{13} d_{21} d_{32}-d_{13} d_{22} d_{31}\right. \\
& \left.-d_{11} d_{23} d_{32}-d_{12} d_{21} d_{33}\right)>0,
\end{aligned}
$$

or

$$
\begin{aligned}
& H_{1}>0, \\
& H_{2}>0, \\
& H_{3}>0,
\end{aligned}
$$

where $H_{i}(i=1,2,3)$ denote the coefficients of ability of synchronization stability. Only when the structural parameters of the system meet Equation (31), can the synchronous states of the system be stable.

Generally in engineering, the parameters of induction motors are selected as the same, that is,

$$
k_{i i} \approx k_{0}, T_{\mathrm{e} 1} \approx T_{\mathrm{e} 2} \approx T_{\mathrm{e} 3} \approx T_{\mathrm{e} 4} \quad(i=1,2,3,4) .
$$

Introducing the following new symbols,

$$
\begin{aligned}
& p_{12}=\eta_{1} \eta_{2} W_{\mathrm{cc} 12} \cos \left(2 \bar{\alpha}_{10}+\theta_{\mathrm{c} 12}\right), \\
& p_{23}=\eta_{2} \eta_{3} W_{\mathrm{cc} 23} \cos \left(2 \bar{\alpha}_{20}+\theta_{\mathrm{c} 23}\right), \\
& p_{34}=\eta_{3} \eta_{4} W_{\mathrm{cc} 34} \cos \left(2 \bar{\alpha}_{30}+\theta_{\mathrm{c} 34}\right), \\
& p_{14}=\eta_{1} \eta_{4} W_{\mathrm{cc} 14} \cos \left(2 \bar{\alpha}_{10}+2 \bar{\alpha}_{20}+2 \bar{\alpha}_{30}+\theta_{\mathrm{c} 14}\right), \\
& p_{13}=\eta_{1} \eta_{3} W_{\mathrm{cc} 13} \cos \left(2 \bar{\alpha}_{10}+2 \bar{\alpha}_{20}+\theta_{\mathrm{c} 13}\right), \\
& p_{24}=\eta_{2} \eta_{4} W_{\mathrm{cc} 24} \cos \left(2 \bar{\alpha}_{20}+2 \bar{\alpha}_{30}+\theta_{\mathrm{c} 24}\right) .
\end{aligned}
$$

Considering Equation (30), we have

$$
\begin{aligned}
& P_{12}>0, \\
& P_{23}>0, \\
& P_{34}>0, \\
& P_{14}>0, \\
& P_{13}>0, \\
& P_{24}>0 .
\end{aligned}
$$

Inserting Equation (32) into Equation (29) and considering Equation (34), the following formulae are deduced:

$$
\begin{aligned}
& a=-d_{11}-d_{22}-d_{33}=\left(\omega_{\mathrm{m} 0}^{*} / k_{0}\right) \\
& \cdot\left(p_{12}+p_{23}+p_{13}+p_{24}+p_{14}+p_{34}\right)>0 \\
e= & \frac{\omega_{\mathrm{m} 0}^{* 3}}{2 k_{0}^{3}}\left(p_{12} p_{23} p_{14}+p_{12} p_{23} p_{24}+p_{12} p_{24} p_{34}+p_{12} p_{13} p_{34}\right. \\
& +p_{12} p_{13} p_{14}+p_{12} p_{13} p_{24}+p_{13} p_{23} p_{34}+p_{13} p_{23} p_{14} \\
& +p_{13} p_{23} p_{24}+p_{13} p_{24} p_{34}+p_{13} p_{24} p_{14}+p_{14} p_{23} p_{34} \\
& \left.+p_{14} p_{23} p_{24}+p_{14} p_{24} p_{34}+p_{12} p_{23} p_{34}+p_{12} p_{34} p_{14}\right)>0
\end{aligned}
$$

$$
\begin{aligned}
a b-e= & \frac{\omega_{\mathrm{m} 0}^{* 3}}{4 k_{0}^{3}}\left(3 p_{14} p_{24}^{2}+4 p_{23} p_{14}^{2}+3 p_{12} p_{24}^{2}+4 p_{13} p_{24}^{2}\right. \\
& +4 p_{24} p_{13}^{2}+3 p_{34} p_{13}^{2}+3 p_{14} p_{13}^{2}+3 p_{34} p_{14}^{2} \\
& +3 p_{12} p_{14}^{2}+3 p_{13} p_{14}^{2}+3 p_{24} p_{14}^{2}+8 p_{12} p_{23} p_{34} \\
& +8 p_{12} p_{23} p_{14}+9 p_{12} p_{24} p_{14}+8 p_{12} p_{13} p_{34} \\
& +7 p_{12} p_{13} p_{14}+8 p_{12} p_{13} p_{24}+7 p_{13} p_{23} p_{34} \\
& +8 p_{13} p_{23} p_{14}+8 p_{13} p_{23} p_{24}+8 p_{13} p_{24} p_{34} \\
& +8 p_{13} p_{24} p_{14}+8 p_{14} p_{23} p_{34}+8 p_{14} p_{23} p_{24} \\
& +7 p_{14} p_{24} p_{34}+9 p_{14} p_{13} p_{34}+8 p_{12} p_{34} p_{14} \\
& +3 p_{23}^{2} p_{34}+4 p_{23}^{2} p_{14}+3 p_{23}^{2} p_{24}+3 p_{23}^{2} p_{13} \\
& +3 p_{23}^{2} p_{12}+3 p_{24}^{2} p_{23}+3 p_{24}^{2} p_{34}+4 p_{34}^{2} p_{12} \\
& +3 p_{34}^{2} p_{13}+3 p_{34}^{2} p_{14}+3 p_{34}^{2} p_{23}+3 p_{34}^{2} p_{24} \\
& +4 p_{12}^{2} p_{14}+3 p_{12}^{2} p_{14}+3 p_{12}^{2} p_{24}+3 p_{12}^{2} p_{13} \\
& +3 p_{12}^{2} p_{23}+3 p_{13}^{2} p_{12}+3 p_{13}^{2} p_{23}+9 p_{23} p_{24} p_{34} \\
& \left.+9 p_{12} p_{13} p_{23}+7 p_{12} p_{23} p_{24}+8 p_{12} p_{24} p_{34}\right)>0
\end{aligned}
$$

Considering the stability criterion (Equation (31)) and Equations (35)-(37), we can achieve stable intervals of phase differences among four vibrators as follows:

$$
\begin{aligned}
& 2 \bar{\alpha}_{10}+\theta_{\mathrm{c} 12} \in\left(-\frac{\pi}{2}, \frac{\pi}{2}\right), \\
& 2 \bar{\alpha}_{20}+\theta_{\mathrm{c} 23} \in\left(-\frac{\pi}{2}, \frac{\pi}{2}\right), \\
& 2 \bar{\alpha}_{30}+\theta_{\mathrm{c} 34} \in\left(-\frac{\pi}{2}, \frac{\pi}{2}\right), \\
& 2 \bar{\alpha}_{10}+2 \bar{\alpha}_{20}+2 \bar{\alpha}_{30}+\theta_{\mathrm{c} 14} \in\left(-\frac{\pi}{2}, \frac{\pi}{2}\right), \\
& 2 \bar{\alpha}_{10}+2 \bar{\alpha}_{20}+\theta_{\mathrm{c} 13} \in\left(-\frac{\pi}{2}, \frac{\pi}{2}\right), \\
& 2 \bar{\alpha}_{20}+2 \bar{\alpha}_{30}+\theta_{\mathrm{c} 24} \in\left(-\frac{\pi}{2}, \frac{\pi}{2}\right) .
\end{aligned}
$$

\section{Numerical Characteristics Analyses}

Based on the above deduced theoretical analytical results on criteria of synchronization and stability of four vibrators, we 
go a step further to analyze the coupling characteristics of the vibrating system in this section by numeric.

It is assumed that four motors are the same and the corresponding parameters are as follows: $M=1430 \mathrm{kG}$, $m_{0}=10 \mathrm{kG}, \quad J=1750 \mathrm{kG} \cdot \mathrm{m}^{2}, \quad k_{\psi}=20000 \mathrm{kN} / \mathrm{m}\left(\mu_{\psi}=\right.$ $0.95), f_{\psi}=3.37 \mathrm{kNs} / \operatorname{rad}\left(\xi_{n x}=\xi_{n y}=\xi_{n \psi}=0.07\right), k_{x}=k_{y}=$ $23000 \mathrm{kN} / \mathrm{m}\left(\mu_{x}=\mu_{y}=0.95\right)$, and $f_{x}=f_{y}=3.83 \mathrm{kNs} / \mathrm{m}$. The type of four motors is three-phase squirrel-cage $(380 \mathrm{~V}$, $50 \mathrm{~Hz}, 6$-pole, and $\Delta$-connected); the parameters $(0.3 \mathrm{~kW}$, rated speed $980 \mathrm{r} / \mathrm{min}$ ) are the stator resistance $R_{\mathrm{s}}=3.35 \Omega$, the rotor resistance $R_{\mathrm{r}}=3.4 \Omega$, the stator inductance $L_{\mathrm{s}}=170 \mathrm{mH}$, the mutual inductance $L_{\mathrm{s}}=164 \mathrm{mH}$, and $f_{1}=f_{2}=f_{3}=f_{4}=0.05$. The natural frequencies are defined as

$$
\begin{aligned}
& \omega_{x}=\sqrt{\frac{k_{x}}{M}}, \\
& \omega_{y}=\sqrt{\frac{k_{y}}{M}}, \\
& \omega_{\psi}=\sqrt{\frac{k_{\psi}}{J}} .
\end{aligned}
$$

Because the stiffnesses of springs in $x$ and $y$ directions are identical, the natural frequencies in these two directions are the same, i.e., $\omega_{x}=\omega_{y}=126.8 \approx 127 \mathrm{rad} / \mathrm{s}$, and $\omega_{\psi}=$ $106.9 \approx 107 \mathrm{rad} / \mathrm{s}$ means the natural frequency in $\psi \mathrm{di}-$ rection. Thus resonant regions of the vibrating system are divided into three ranges: regions $\mathrm{I}\left(\omega_{\mathrm{m} 0}<\omega_{\psi}\right)$, $\operatorname{II}\left(\omega_{\psi}<\right.$ $\left.\omega_{\mathrm{m} 0}<\omega_{x}\right)$, and III $\left(\omega_{\mathrm{m} 0}>\omega_{x}\right)$. It should be noted that in the following numeric characteristic analyses, we obtain the rounding values of $\omega_{x}, \omega_{y}$, and $\omega_{\psi}$ in order to only discuss and describe conveniently, i.e., $\omega_{x}=\omega_{y} \approx 127 \mathrm{rad} / \mathrm{s}$ and $\omega_{\psi} \approx 107 \mathrm{rad} / \mathrm{s}$, which cannot influence the synchronization of the four vibrators and also not change the physical configuration of the system.

4.1. Synchronization Criterion. As shown in Section 3, $\tau_{\text {cij }}\left(\bar{\alpha}_{1}, \bar{\alpha}_{2}, \bar{\alpha}_{3}\right)$ is the function of $\alpha_{1}, \alpha_{2}$, and $\alpha_{3}$. Figure 2 shows that values of $\tau_{\text {cij max }}$ for the different $\eta_{i}(i=1,2,3,4)$, in which maxima of $\tau_{c i j \max }$ are achieved by $\omega_{x}=127 \mathrm{rad} / \mathrm{s}$, and $\tau_{c i j \max }$ under the condition of $\eta_{1}=\eta_{2}=\eta_{3}=\eta_{4}=1$ is larger than the others, and in this case, vibrators are easiest to implement synchronization.

The parameter $\zeta_{i j}$ denotes the coefficient of synchronization ability (CSA) and their expressions are given in Equation (20). Figure 3 shows the CSAs among four vibrators for the different $\eta_{i}$. In Figure 3(a), we can see that $\zeta_{12}=\zeta_{23}=\zeta_{34}=\zeta_{41}>\zeta_{13}=\zeta_{24}$ due to complete symmetry of the system; Figures 3(b) and 3(c) have $\zeta_{12}=\zeta_{23}>\zeta_{34}>$ $\zeta_{41}>\zeta_{13}>\zeta_{24}$ and $\zeta_{12}>\zeta_{23}>\zeta_{41}>\zeta_{24}>\zeta_{13}>\zeta_{34}$, respectively, which indicate the important influence of $\eta_{i}$ to synchronization. It should be noted here that the derivation method of $\tau_{c i j \max }$ and $\zeta_{i j}(i j=13,24)$ is similar to that of Equations (8)-(10), and hence, it is not dwelt on again. Additionally, CSAs in all conditions decrease as $\omega_{\mathrm{m} 0}$ increases in region $\operatorname{III}\left(\omega_{\mathrm{m} 0}>\omega_{x}\right)$.
4.2. Stable Phase Differences. From Figure 4(a), we can find that the phase differences in region $\mathrm{I}\left(\omega_{\mathrm{m} 0}<\omega_{\psi}\right)$ are the same as that in region $\operatorname{II}\left(\omega_{\psi}<\omega_{\mathrm{m} 0}<\omega_{x}\right)$. Due to the fact of the small difference value between $\omega_{x}$ and $\omega_{\psi}$ in engineering, we may combine regions I and II into one common region, which is defined as the subresonant region with respect to $\omega_{x}$, while region III is defined as the superresonant region for $\omega_{x}$. Here L1 means the subresonant region and L2 refers to the superresonant region. Dashed line A means the simulation point of the subresonant region in next section, and 74 means $\omega_{\mathrm{m} 0}=74 \mathrm{rad} / \mathrm{s}$. As such dashed line B means the simulation point of the superresonant region and 198 for $\omega_{\mathrm{m} 0}=198 \mathrm{rad} / \mathrm{s}$. The validity of these calculated values will be verified in next section of computer simulations.

It can be seen that from Figure 4(a), in the case of stabilization, the phase differences are all close to $0^{\circ}$ in the subresonance region, while in the superresonance region, the phase differences are near $90^{\circ}$ and $180^{\circ}$, which denotes the existence of diversity of nonlinear system due to the existence of double equilibrium points [28].

The vibrators 1, 2, and 3 are the same, and the mass of the vibrator 4 is changed, and the stable phase difference curves of Figure 4(b) are obtained. The phase differences are near to $0^{\circ}$ in the subresonance region; while in the superresonance region, there are two groups of stable phase differences among vibrators: $2 \alpha_{1-1}=104^{\circ}, 2 \alpha_{1-2}=151^{\circ}, 2 \alpha_{2-1}=108^{\circ}$, $2 \alpha_{2-2}=158^{\circ}, 2 \alpha_{3-1}=72^{\circ}, 2 \alpha_{3-2}=-158^{\circ}$. While in the nearresonance region, the transition value of the phase difference appears.

The vibrators 1 and 2 are the same, and masses of the vibrators 3 and 4 are changed; the stable phase difference curves of Figure 4(c) are obtained; the phase differences are in the vicinity of $0^{\circ}$ in the subresonance region, while in the superresonance region, there are two stable phase differences: $2 \alpha_{1-1}=112^{\circ}, 2 \alpha_{1-2}=153^{\circ}, 2 \alpha_{2-1}=104^{\circ}, 2 \alpha_{2-2}=162^{\circ}$, $2 \alpha_{3-1}=54^{\circ}, 2 \alpha_{3-2}=-144^{\circ}$. In this case, the transition value of the phase differences also appears in the near-resonance region.

\section{Computer Simulations}

In this section, the vibrating system is simulated by using the fourth-order Runge-Kutta routine. Here, it is assumed that the mass of the standard vibrator is $m_{0}=10 \mathrm{kG}$, and the eccentric radii of four vibrators are same. Meanwhile, in the subresonant region, $k_{x}=23000 \mathrm{kN} / \mathrm{m}$ and in the superresonant region, $k_{x}=6240 \mathrm{kN} / \mathrm{m}$; other parameters are the same as those in the previous section. Using quantitative analysis of simulations to verify qualitative analysis of characteristic analysis, we change spring stiffness to implement simulations of two regions. Thus, we need to introduce a new variable; it is the frequency ratio $z$, which can facilitate to derive $\omega_{\mathrm{m} 0}$ of the simulation point through it.

Equation (40) denotes the frequency ratio; we substitute $k_{x}=23000 \mathrm{kN} / \mathrm{m}$ into Equation (39) and obtain a new natural frequency of the point $A$, that is $\omega_{n}^{*}=178 \mathrm{rad} / \mathrm{s}$. Simultaneously, based on the rotational speed in simulation $\left(\omega_{\mathrm{SA}}=103 \mathrm{rad} / \mathrm{s}\right)$ and Equation (40), the frequency ratio of the simulation point $\mathrm{A}$ is obtained, that is $z=0.58$. 


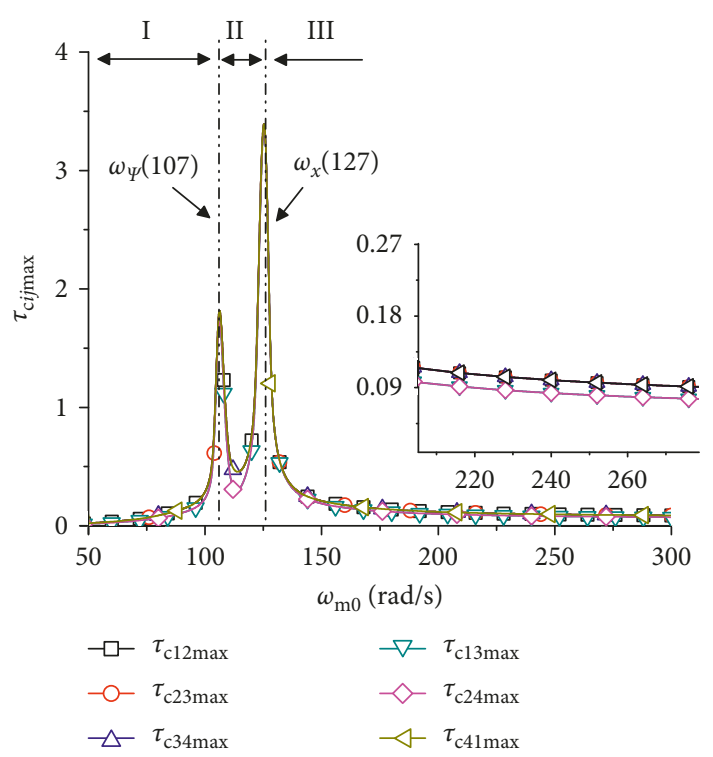

(a)

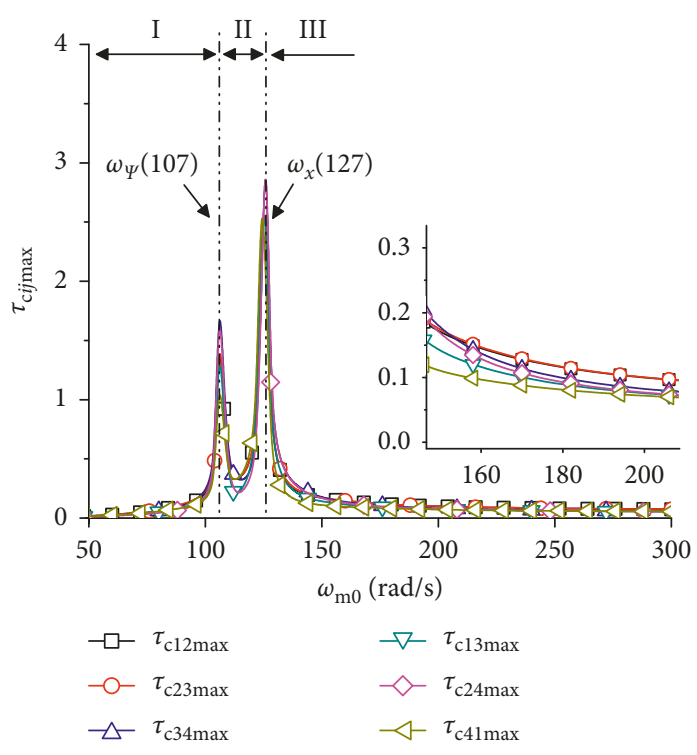

(b)

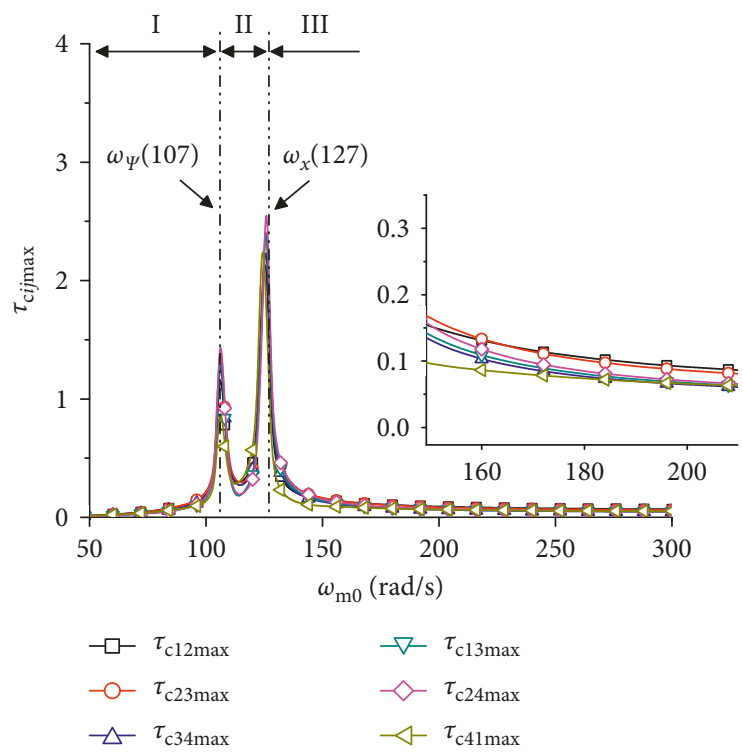

(c)

FIGURE 2: $\tau_{\text {cijmax }}$ in different $\eta_{i}(i=1,2,3,4)$. (a) $\eta_{1}=\eta_{2}=\eta_{3}=\eta_{4}=1$; (b) $\eta_{1}=\eta_{2}=\eta_{3}=1, \eta_{4}=0.5$; (c) $\eta_{1}=\eta_{2}=1, \eta_{3}=0.75, \eta_{4}=0.5$.

Through the frequency ratio, we can deduce that the operating frequency of the simulation point $\mathrm{A}$ is $\omega_{\mathrm{m} 0}=$ $74 \mathrm{rad} / \mathrm{s}$. In the same way, the frequency ratio and operating frequency of the simulation point of $\mathrm{B}$ are $z=1.56$ and $\omega_{\mathrm{m} 0}=198 \mathrm{rad} / \mathrm{s}$, when we consider $k_{x}=6240 \mathrm{kN} / \mathrm{m}$.

$$
z=\frac{\omega_{\mathrm{m} 0}}{\omega_{n}}
$$

In other words, the simulation of subresonant region is in the condition of $z=0.58$, corresponding to the simulation point A of Figure 4 and that of superresonant region is selected for $z=1.56$ corresponding to the simulation point B of Figure 4.

\subsection{Superresonant Region (Simulation Point B) Simulations}

5.1.1. Simulation for $\eta_{1}=\eta_{2}=\eta_{3}=\eta_{4}=1$. Here, masses of four vibrators are identical, i.e., $m_{i}=m_{0}=10 \mathrm{kG}(i=1,2,3$, 4). In Figure 5, we can see the following results: the angular acceleration of motors is identical when the four motors are in the starting process; therefore, the phase differences are near to $0^{\circ}$. But the responses of the system are aroused when the angular velocity of motors reaches resonance regions of the vibrating system. Until the angular velocity reaches stable and four vibrators can operate synchronously, the high frequency vibration is aroused, and in this moment, the synchronous rotational velocity of motors is $983 \mathrm{r} / \mathrm{min}$, and 


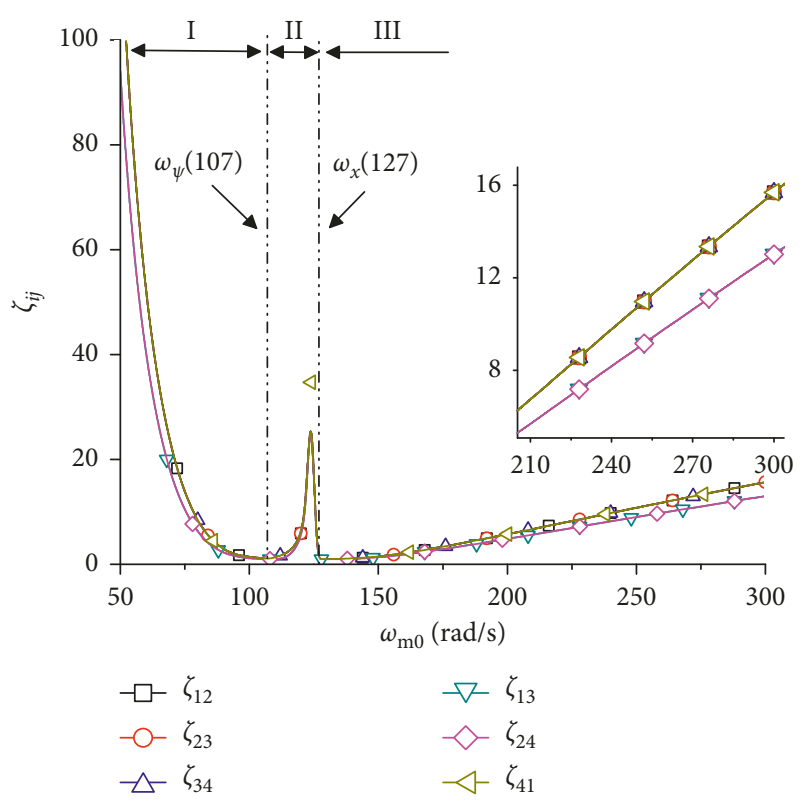

(a)

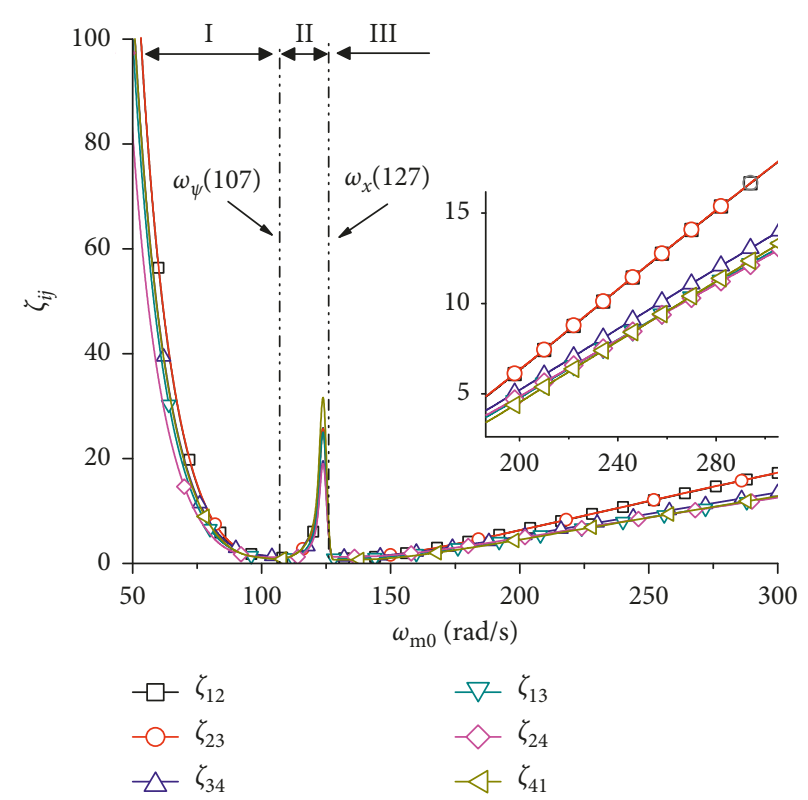

(b)

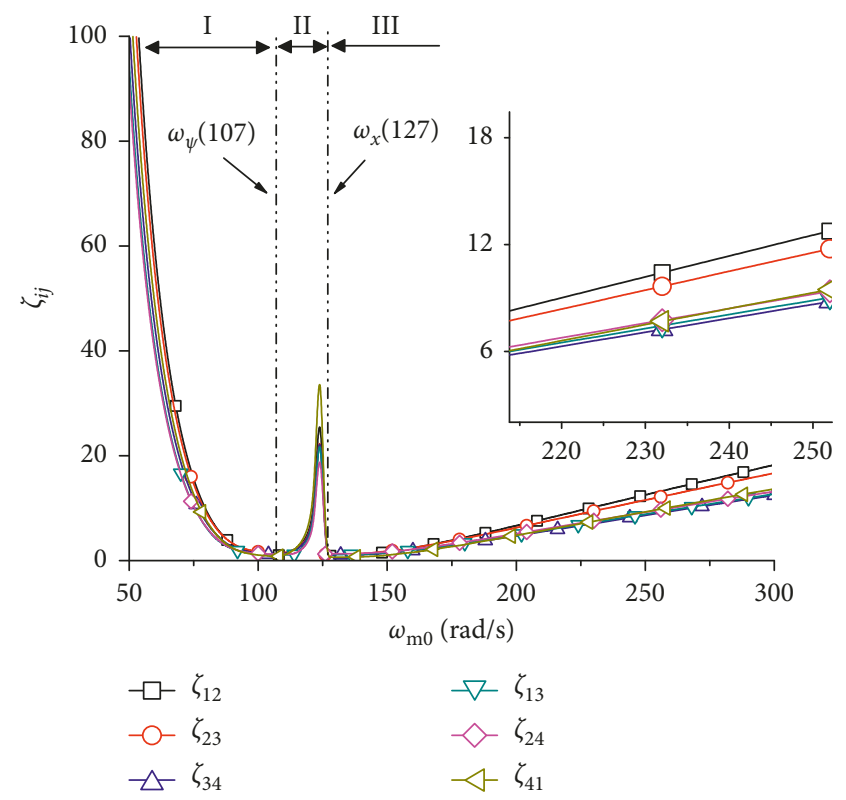

(c)

FIGURE 3: CGDSs among four vibrators in different $\eta_{i}(i=1,2,3,4)$. (a) $\eta_{1}=\eta_{2}=\eta_{3}=\eta_{4}=1$; (b) $\eta_{1}=\eta_{2}=\eta_{3}=1, \eta_{4}=0.5$; (c) $\eta_{1}=\eta_{2}=1, \eta_{3}=0.75, \eta_{4}=0.5$.

the phase differences are near to $180^{\circ}$. In Figures $5(b)-5(d)$, at $20 \mathrm{~s}$, a disturbance of $\pi$ phase applies to the vibrator $2 ; 2 \alpha_{1}$ transforms from $180^{\circ}$ to $90^{\circ}$, as well as $2 \alpha_{2}$ and $2 \alpha_{3}$, which indicates that there are two equilibrium points in the system, and these results agree well with that of simulation point $B$ in Figure 4(a).

In Figure 5(a), at $40 \mathrm{~s}$, we know that the system is back to the steady state and the synchronous rotational velocity of motors changes to $977 \mathrm{r} / \mathrm{min}$. Meanwhile, in Figures 5(b)$5(\mathrm{~d})$, at $40 \mathrm{~s}$, the electricity of the system is switched off, and then $2 \alpha_{1}$ transforms from $90^{\circ}$ to $107.23^{\circ}, 2 \alpha_{2}$ from $90^{\circ}$ to $70.15^{\circ}$, and $2 \alpha_{3}$ from $90^{\circ}$ to $94.86^{\circ}$, which means that the phenomenon of vibratory synchronization transmission occurs in the system.

In Figures 5(e) and 5(f), the resonant responses of the rigid frame in $x, y$, and $\psi$ directions are close to 0 , when the system is back to the steady state, which indicates that the exciting forces of four vibrators are canceled with each other, and the system can still operate synchronously and stably in the superresonant region.

5.1.2. Simulation for $\eta_{1}=\eta_{2}=\eta_{3}=1, \eta_{4}=0.5$. The analysis method in this section is the same as the previous section. 

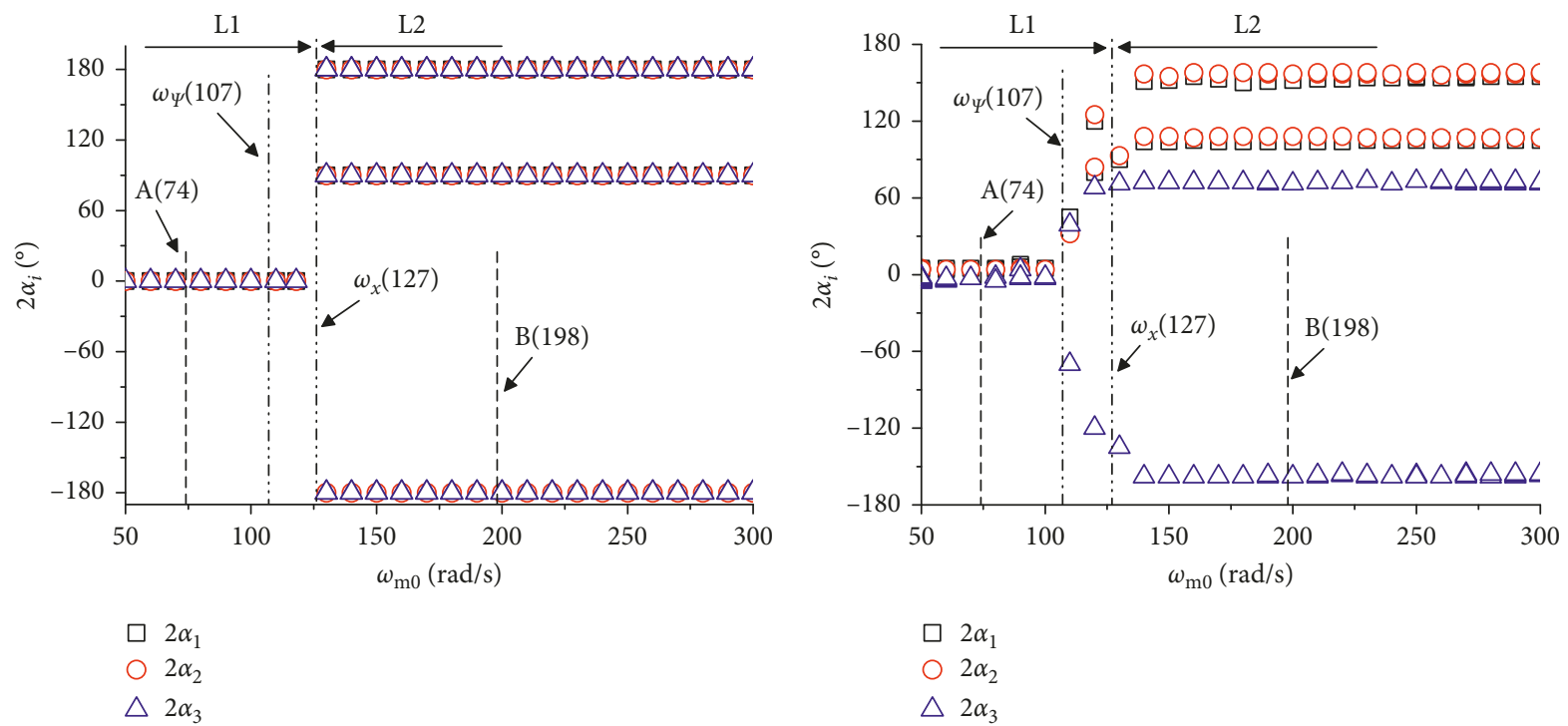

(a)

(b)

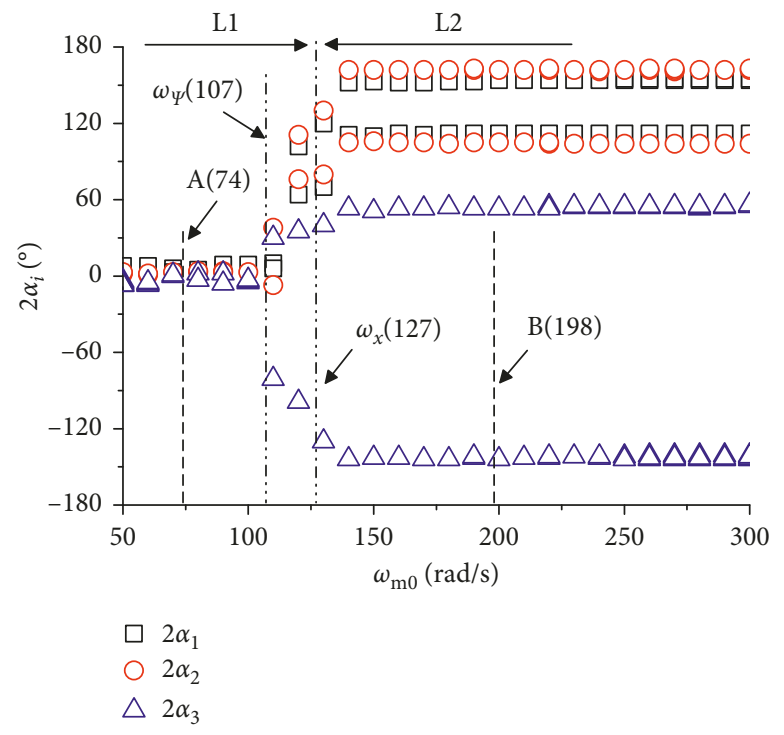

(c)

FiguRE 4: Stable difference-values of phase in the different $\eta_{i}(i=1,2,3,4)$. (a) $\eta_{1}=\eta_{2}=\eta_{3}=\eta_{4}=1$; (b) $\eta_{1}=\eta_{2}=\eta_{3}=1, \eta_{4}=0.5$; (c) $\eta_{1}=\eta_{2}=1, \eta_{3}=0.75, \eta_{4}=0.5$.

Figure 6 shows that the synchronous rotational velocity is $983 \mathrm{r} / \mathrm{min}$, and the phase differences are $2 \alpha_{1}=105^{\circ}$, $2 \alpha_{2}=106^{\circ}, 2 \alpha_{3}=70.6^{\circ}$, when four vibrators operate synchronously. A disturbance of $\pi$ phase applies to the vibrator 2 , when time passes through $20 \mathrm{~s}$ and the system reach another steady state; in this moment, the phase differences change to $2 \alpha_{1}=153^{\circ}, 2 \alpha_{2}=152^{\circ}, 2 \alpha_{3}=-155^{\circ}$. Contrasting the results with simulation point $B$ in Figure $4(\mathrm{~b})$, we can find that they are alike. At $40 \mathrm{~s}$, the electricity of the system is switched off, and we see that the system is back to the steady state from Figure 6(a); in this case, the phase differences are $2 \alpha_{1}=115^{\circ}, 2 \alpha_{2}=96^{\circ}, 2 \alpha_{3}=64^{\circ}$, and the synchronous rotational velocity changes to $978 \mathrm{r} / \mathrm{min}$. These facts show that the vibrating system still can operate by the way of vibratory synchronization transmission.
5.1.3. Simulation for $\eta_{1}=\eta_{2}=1, \eta_{3}=0.75, \eta_{4}=0.5$. Figure 7 shows the following results. The four vibrators operate synchronously, when time reaches about $5 \mathrm{~s}$. At this time, the phase differences are $2 \alpha_{1}=155^{\circ}, 2 \alpha_{2}=158^{\circ}$, $2 \alpha_{3}=-144^{\circ}$ and the synchronous rotational velocity is $983 \mathrm{r} / \mathrm{min}$. Similarly, a disturbance of $\pi$ phase applies to the vibrator 2 , and the phase differences transform to $2 \alpha_{1}=112^{\circ}, 2 \alpha_{2}=103^{\circ}, 2 \alpha_{3}=51^{\circ}$. Reviewing characteristic analysis of Figure 4(c), we can know that the simulation results are line with it. When the electricity of the system is switched off, the system is back to the steady state and still keeps operating synchronously. In this moment, the phase differences are $2 \alpha_{1}=125^{\circ}$, $2 \alpha_{2}=89.85^{\circ}, 2 \alpha_{3}=42.99^{\circ}$ and the synchronous rotational velocity is $978 \mathrm{r} / \mathrm{min}$. 


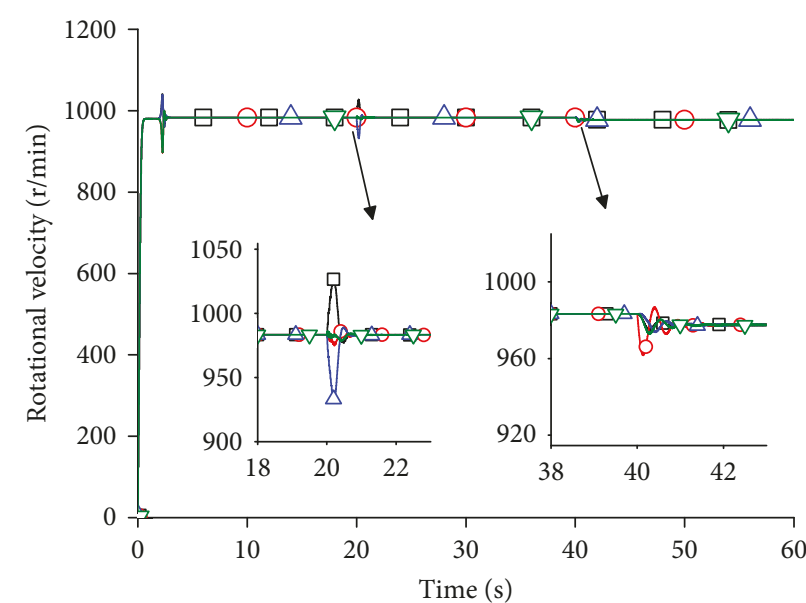

$-\square-$ Motor 1

$-\mathrm{O}-$ Motor 2

$\triangle$ Motor 3

$-\nabla-$ Motor 4

(a)

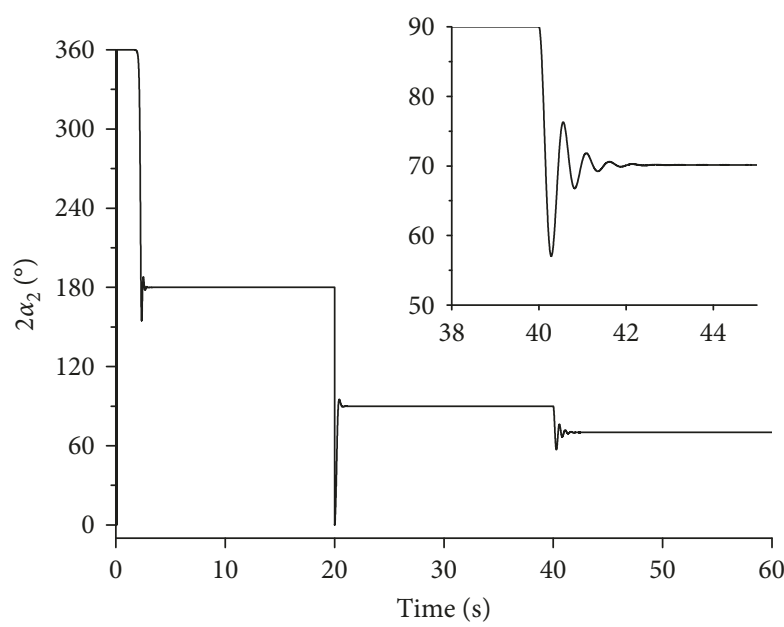

(c)

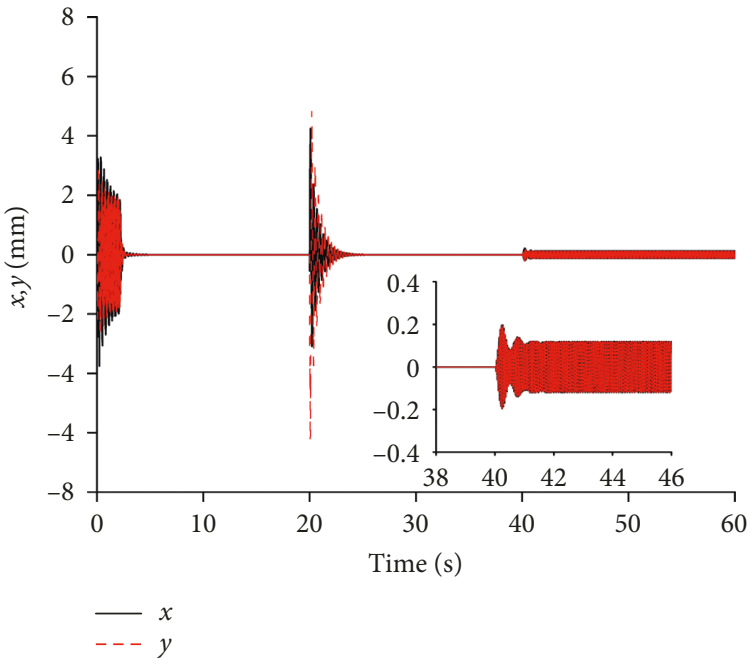

(e)

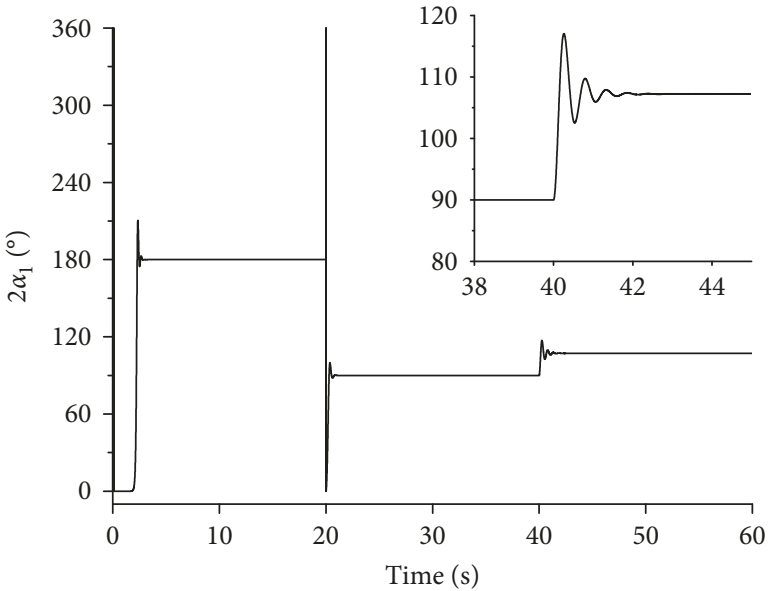

(b)

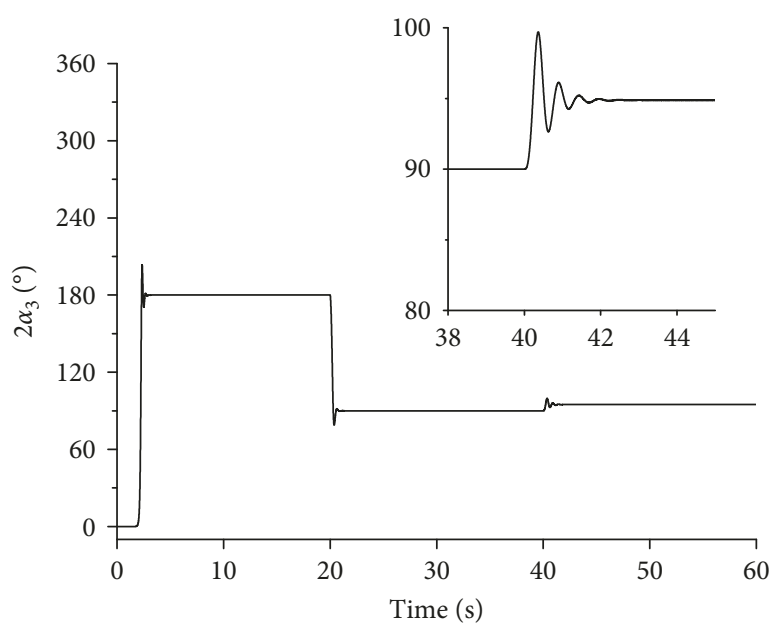

(d)

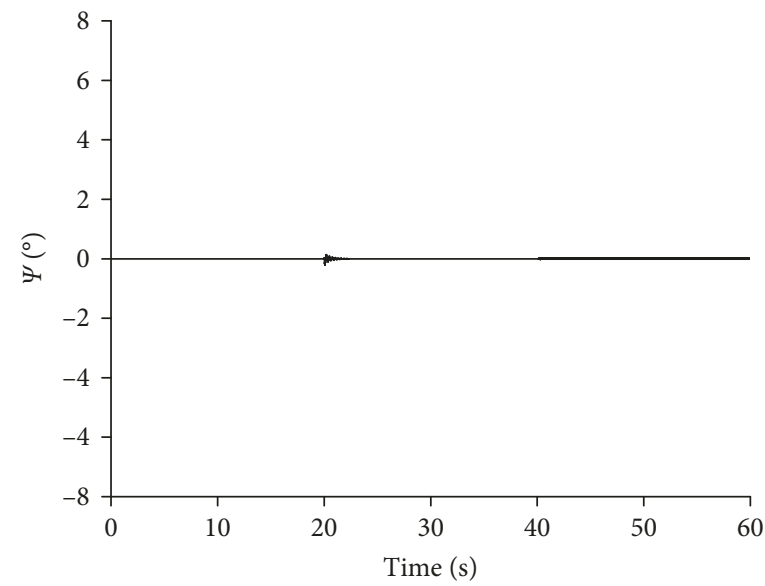

(f)

FiguRE 5: Results of computer simulations for $\eta_{1}=\eta_{2}=\eta_{3}=\eta_{4}=1$. (a) Rotational velocities of the four motors; (b) phase difference between vibrator 1 and 2; (c) phase difference between vibrator 2 and 3; (d) phase difference between vibrator 3 and 4; (e) displacements in $x$ and $y$ directions; (f) displacement in $\psi$ direction. 


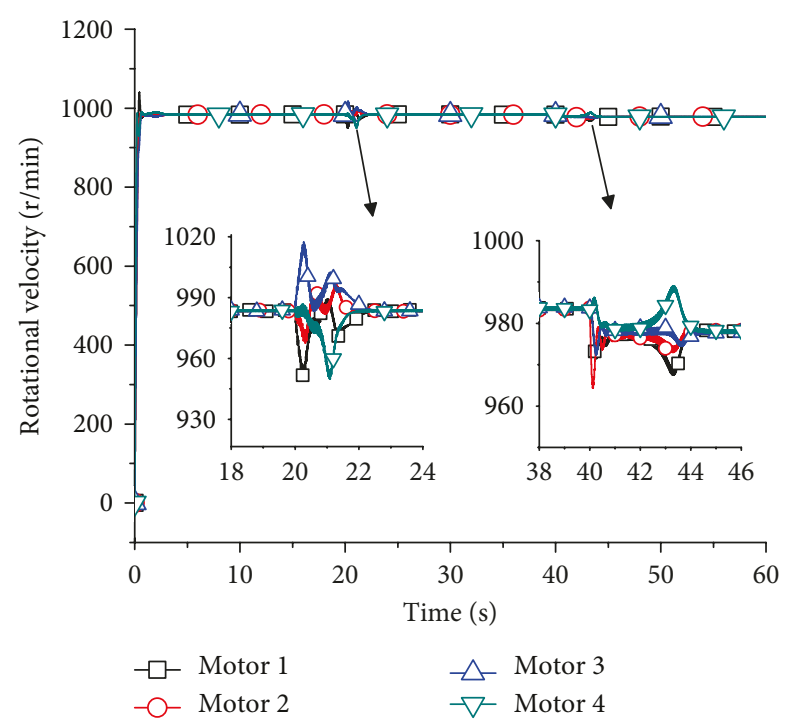

(a)

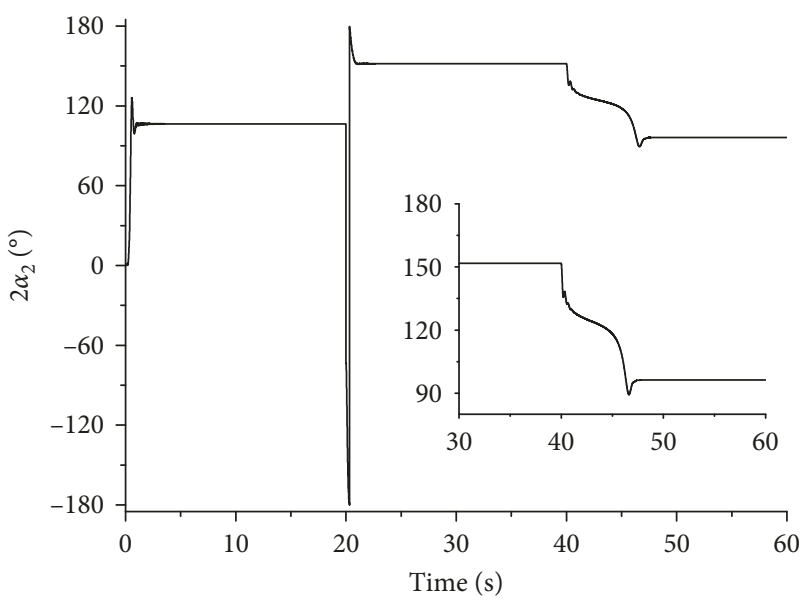

(c)

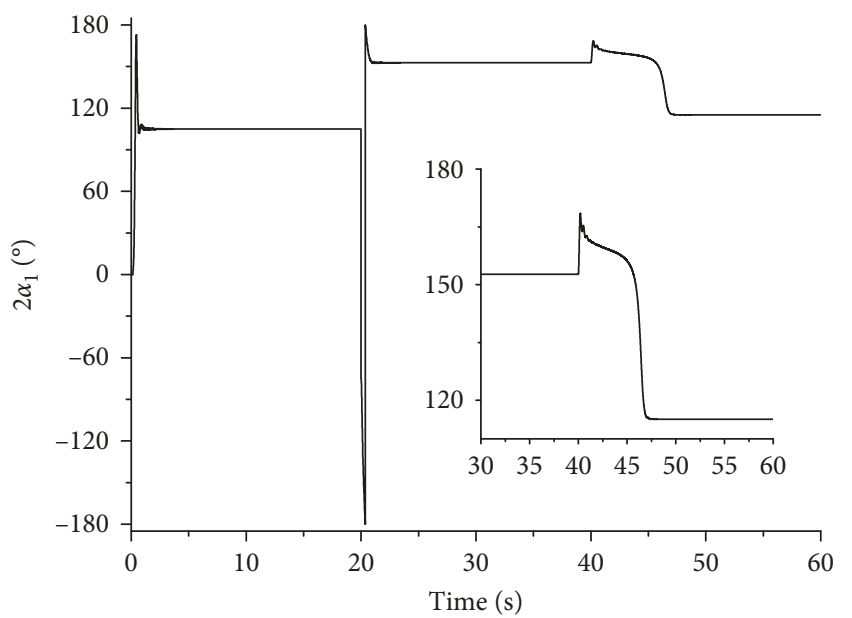

(b)

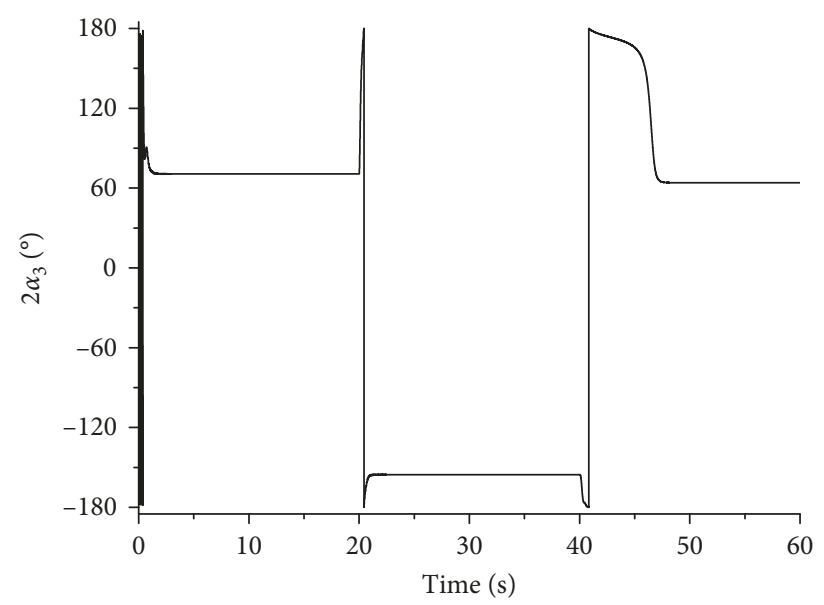

(d)

FIGURE 6: Results of computer simulations for $\eta_{1}=\eta_{2}=\eta_{3}=1, \eta_{4}=0.5$. (a) Rotational velocities of the four motors; (b) phase difference between vibrators 1 and 2; (c) phase difference between vibrators 2 and 3; (d) phase difference between vibrators 3 and 4 .

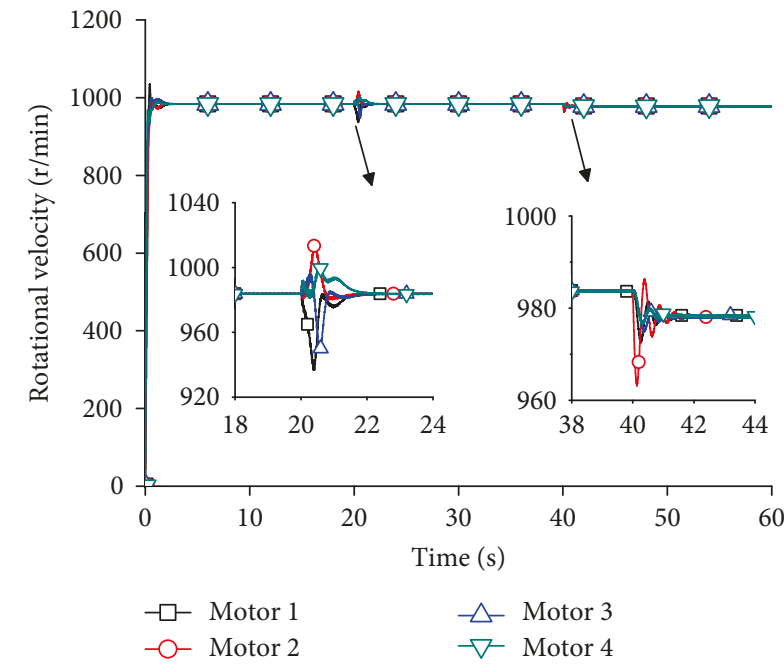

(a)

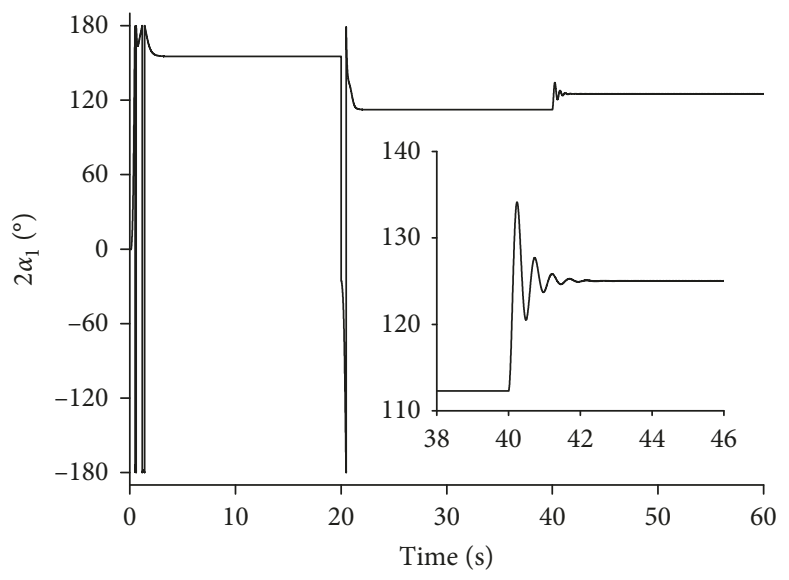

(b)

Figure 7: Continued. 


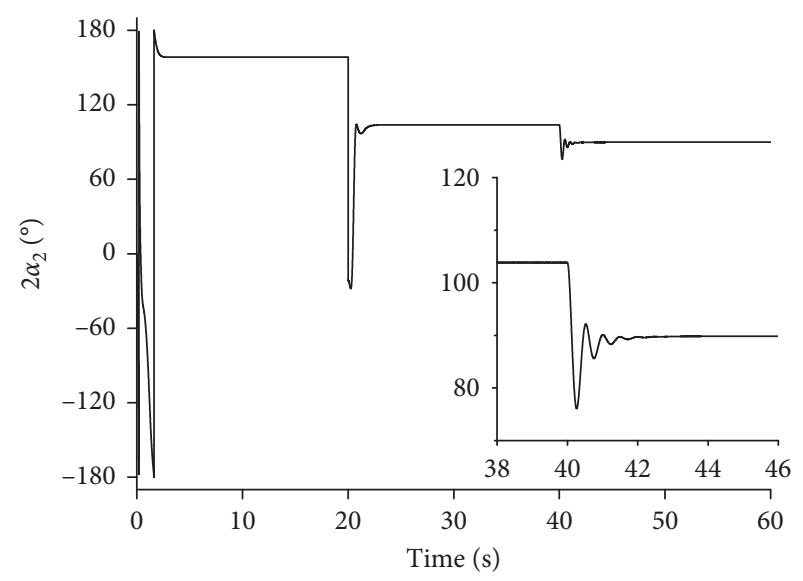

(c)

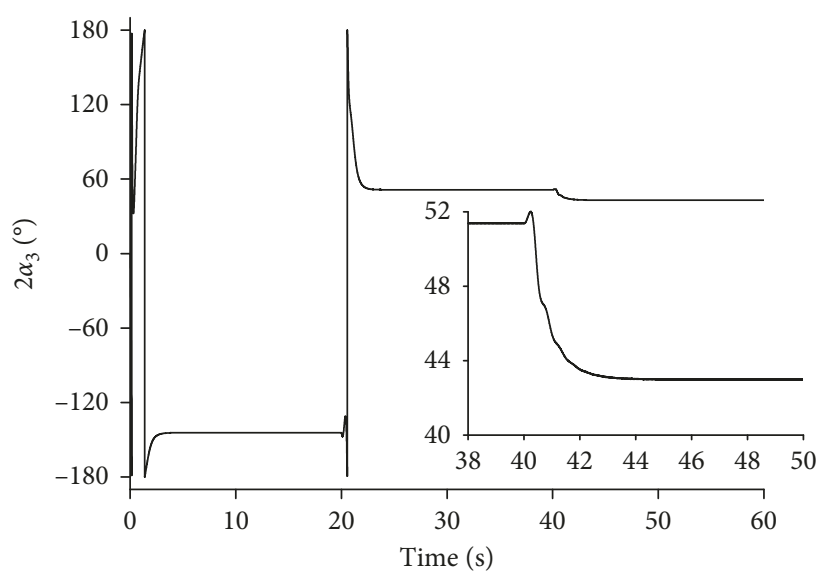

(d)

FIgURE 7: Results of computer simulations for $\eta_{1}=\eta_{2}=1, \eta_{3}=0.75, \eta_{4}=0.5$. (a) Rotational velocities of the four motors; (b) phase difference between vibrators 1 and 2; (c) phase difference between vibrators 2 and 3; (d) phase difference between vibrators 3 and 4 .

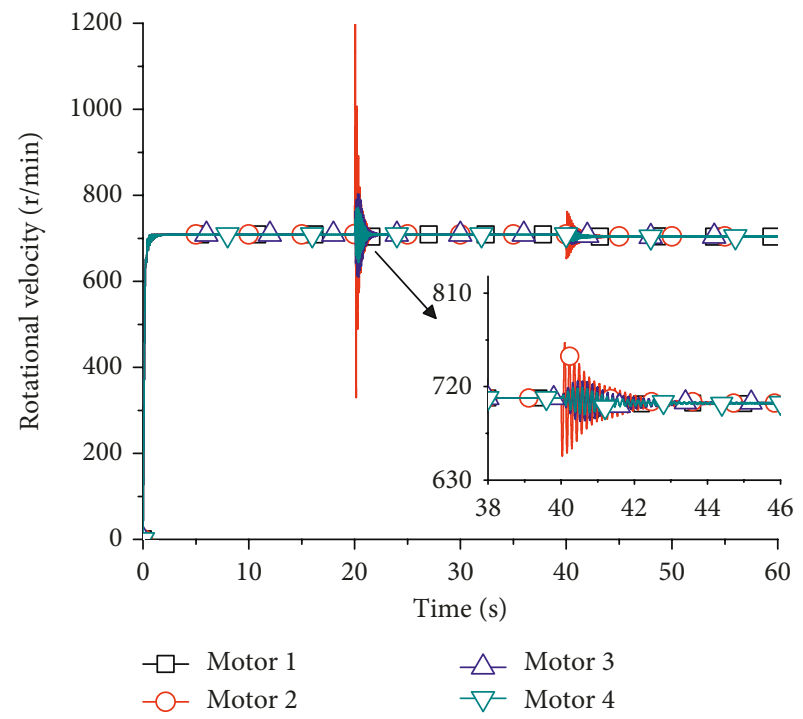

(a)

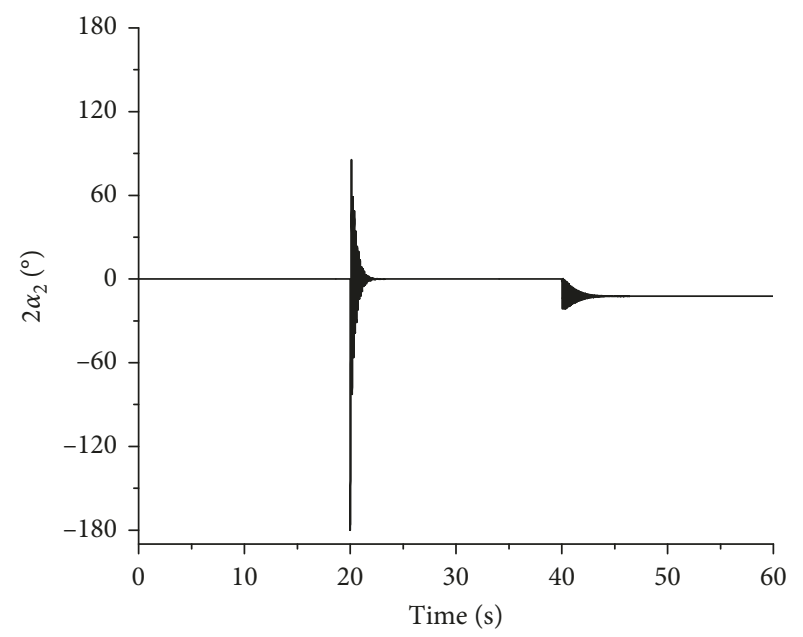

(c)

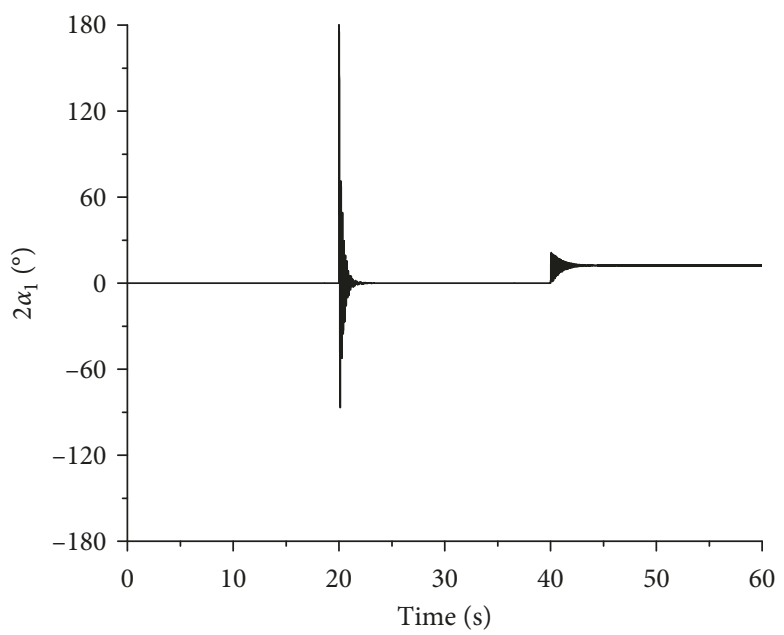

(b)

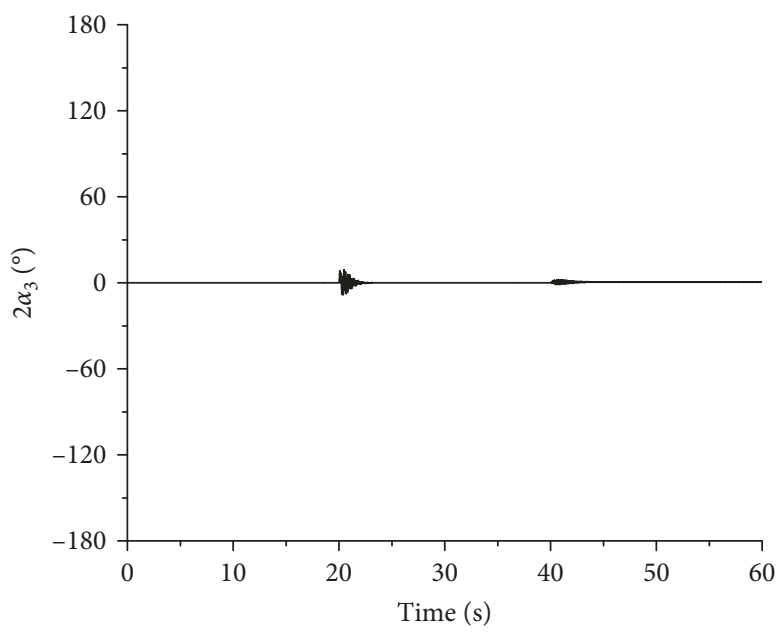

(d)

Figure 8: Continued. 


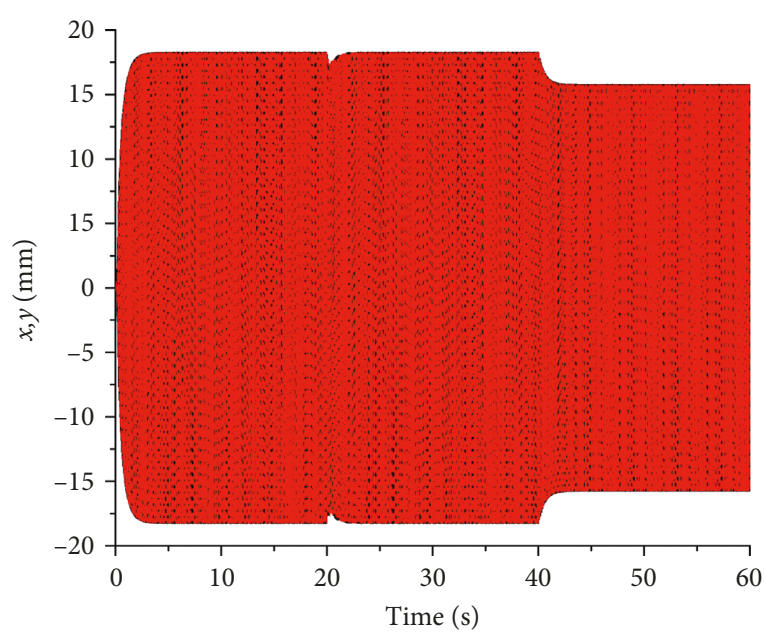

$x$
$---y$

(e)

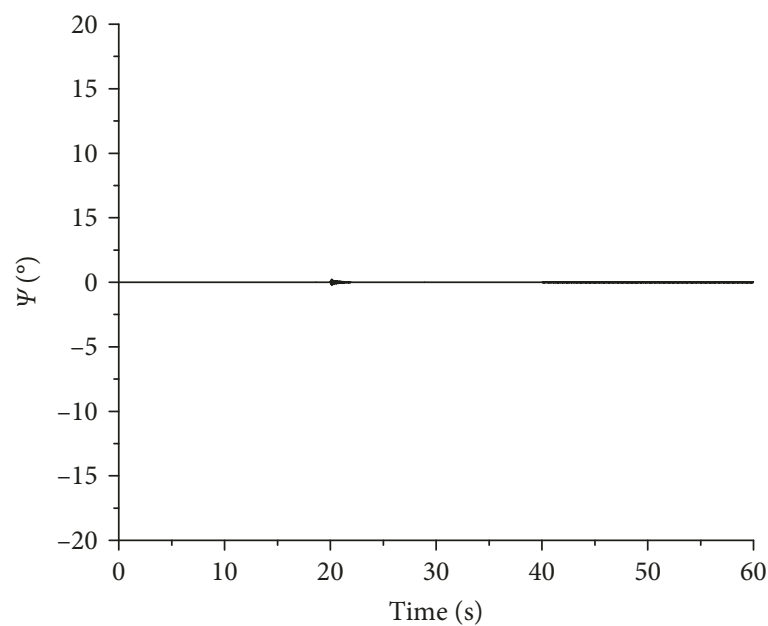

(f)

Figure 8: Results of computer simulations for $\eta_{1}=\eta_{2}=\eta_{3}=\eta_{4}=1$. (a) Rotational velocities of the four motors; (b) phase difference between vibrator 1 and 2; (c) phase difference between vibrator 2 and 3; (d) phase difference between vibrator 3 and 4; (e) displacements in $x$ and $y$ directions; (f) displacement in $\psi$ direction.

TABLE 1: The stable phase differences for $\eta_{1}=\eta_{2}=\eta_{3}=\eta_{4}=1$.

\begin{tabular}{|c|c|c|c|c|}
\hline & & Simulation of point $\mathrm{A}$ & & Characteristic \\
\hline & $\begin{array}{c}\text { Stable phase differences before } \\
\text { disturbance }\end{array}$ & $\begin{array}{c}\text { Stable phase differences after } \\
\text { disturbance }\end{array}$ & $\begin{array}{c}\text { Stable phase differences after } \\
\text { outage }\end{array}$ & $\begin{array}{c}\text { Stable phase } \\
\text { differences }\end{array}$ \\
\hline $2 \alpha_{1}$ & $0^{\circ}$ & $0^{\circ}$ & $12.3^{\circ}$ & $0^{\circ}$ \\
\hline $2 \alpha_{2}$ & $0^{\circ}$ & $0^{\circ}$ & $-12.3^{\circ}$ & $0^{\circ}$ \\
\hline $2 \alpha_{3}$ & $0^{\circ}$ & $0^{\circ}$ & $0.5^{\circ}$ & $0^{\circ}$ \\
\hline
\end{tabular}

*The results of characteristic analysis data from Figure 4(a).

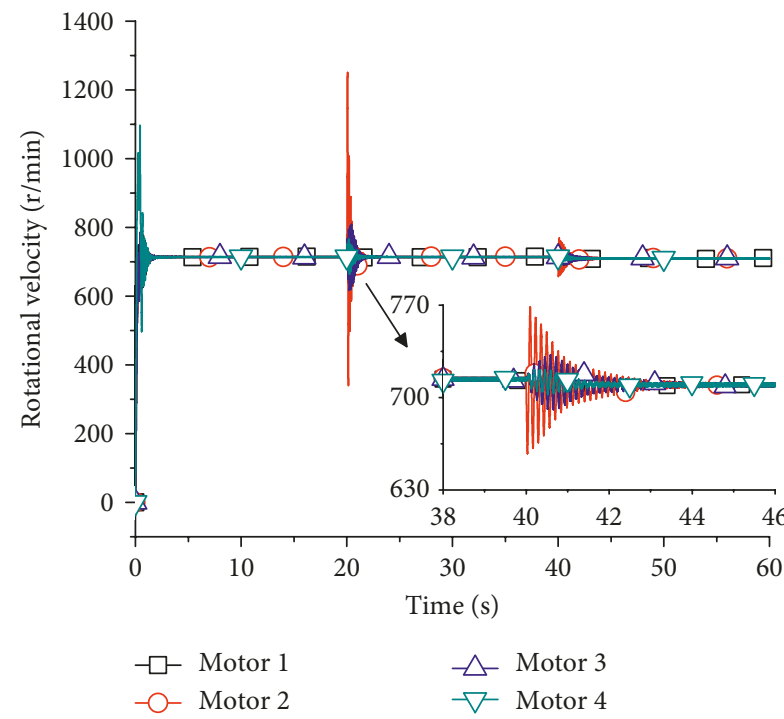

(a)

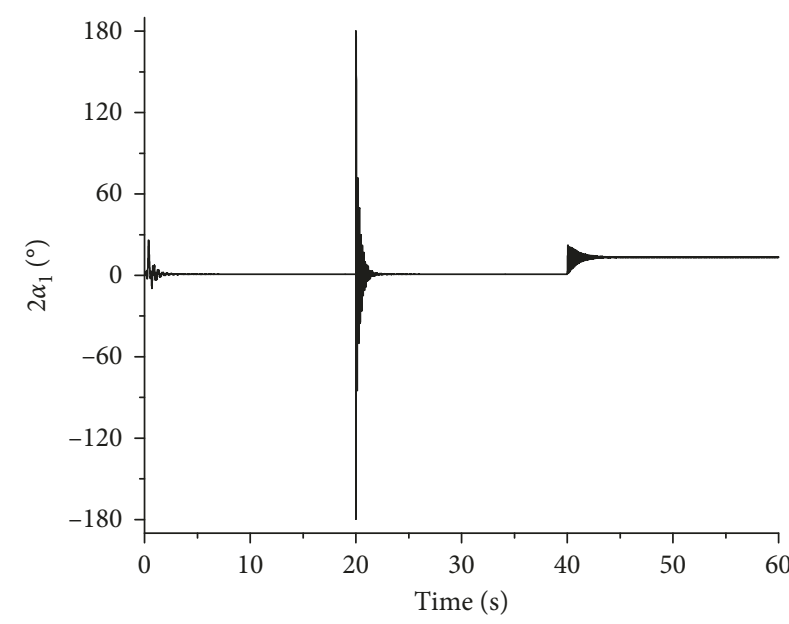

(b)

FIGURE 9: Continued. 


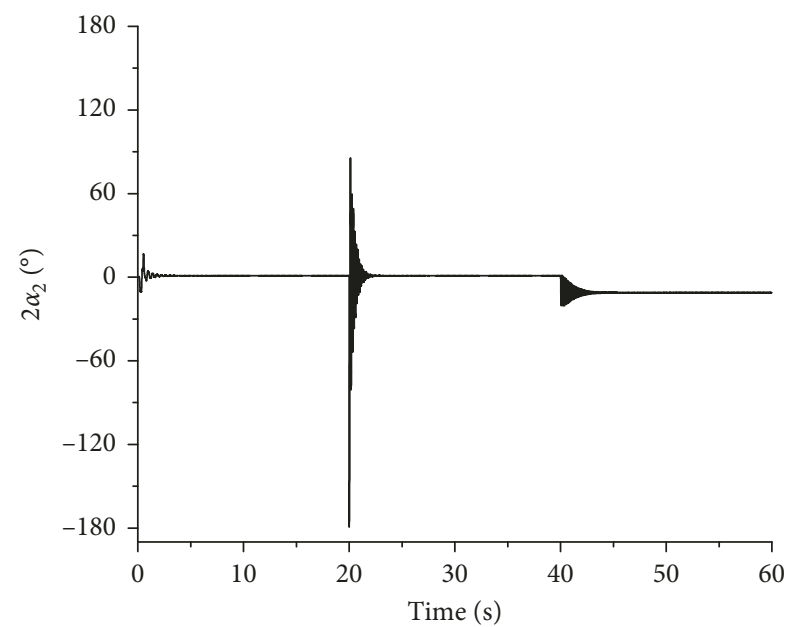

(c)

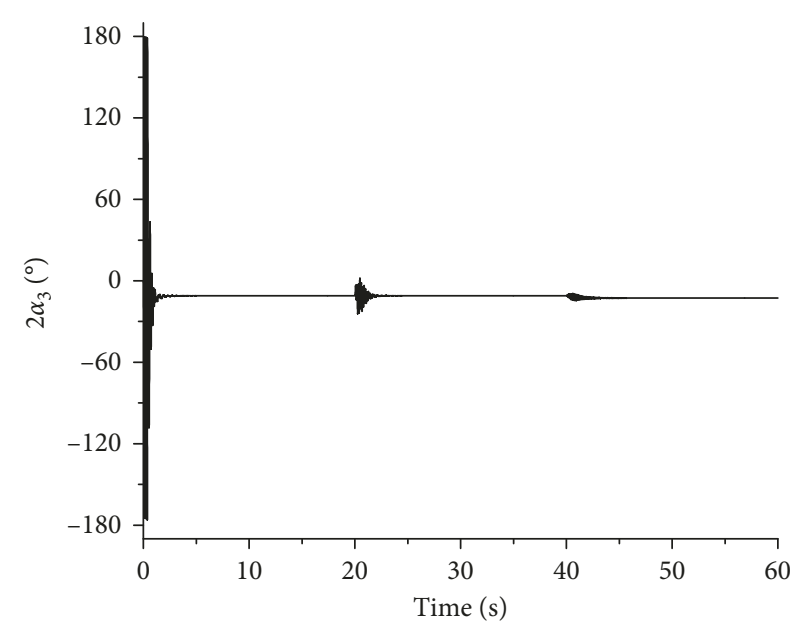

(d)

FIGURE 9: Results of computer simulations for $\eta_{1}=\eta_{2}=\eta_{3}=1, \eta_{4}=0.5$. (a) Rotational velocities of the four motors; (b) phase difference between vibrators 1 and 2; (c) phase difference between vibrators 2 and 3; (d) phase difference between vibrators 3 and 4 .

TABLE 2: The stable phase differences for $\eta_{1}=\eta_{2}=\eta_{3}=1, \eta_{4}=0.5$.

\begin{tabular}{|c|c|c|c|c|}
\hline & & Simulation of point A & & Characteristic \\
\hline & $\begin{array}{c}\text { Stable phase differences before } \\
\text { disturbance }\end{array}$ & $\begin{array}{l}\text { Stable phase differences after } \\
\text { disturbance }\end{array}$ & $\begin{array}{c}\text { Stable phase differences after } \\
\text { outage }\end{array}$ & $\begin{array}{l}\text { Stable phase } \\
\text { differences }\end{array}$ \\
\hline $2 \alpha_{1}$ & $0.8^{\circ}$ & $0.8^{\circ}$ & $13.3^{\circ}$ & $5^{\circ}$ \\
\hline $2 \alpha_{2}$ & $1^{\circ}$ & $1^{\circ}$ & $-11.1^{\circ}$ & $4^{\circ}$ \\
\hline $2 \alpha_{3}$ & $-11^{\circ}$ & $-11^{\circ}$ & $-12.65^{\circ}$ & $-3^{\circ}$ \\
\hline
\end{tabular}

${ }^{*}$ The results of characteristic analysis come from Figure 4(b).

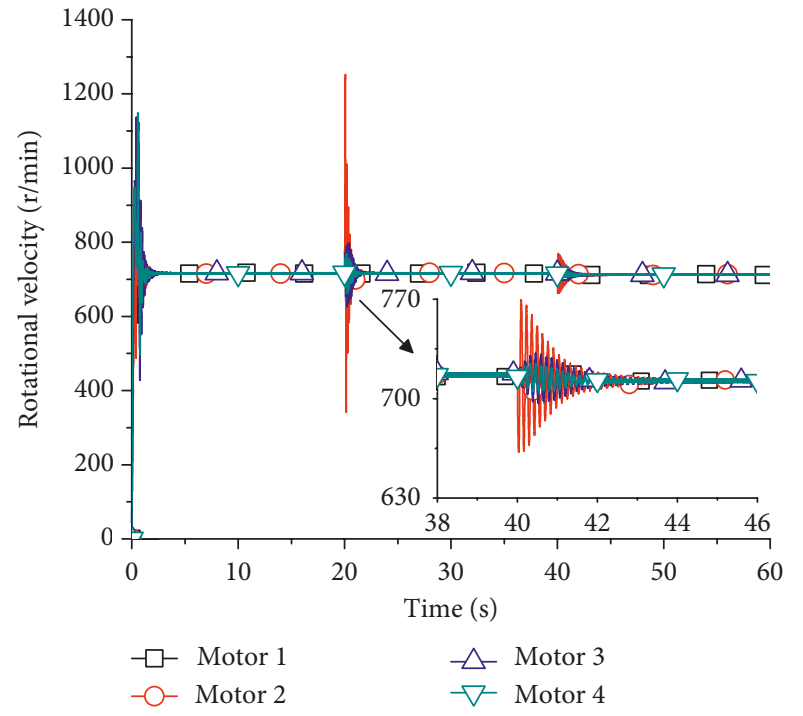

(a)

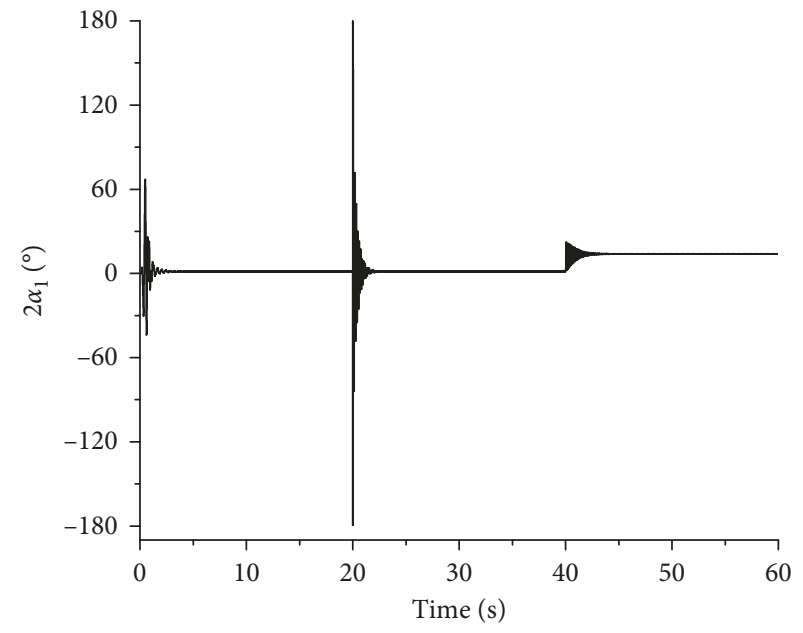

(b)

Figure 10: Continued. 


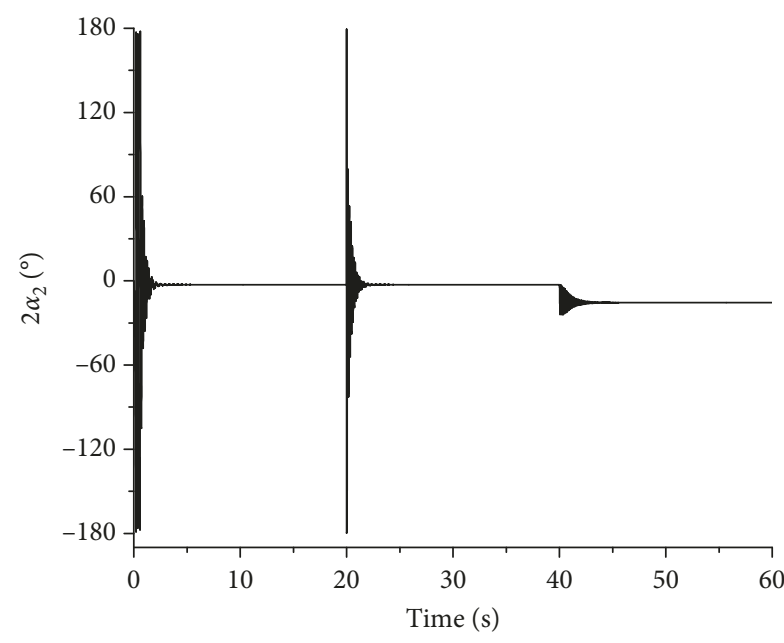

(c)

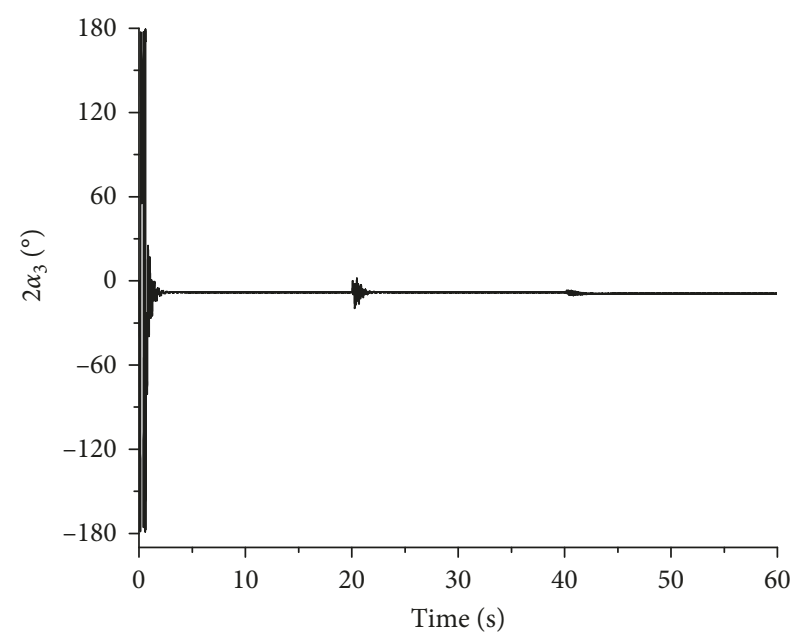

(d)

Figure 10: Results of computer simulations for $\eta_{1}=\eta_{2}=1, \eta_{3}=0.75, \eta_{4}=0.5$. (a) Rotational velocities of the four motors; (b) phase difference between vibrators 1 and 2; (c) phase difference between vibrators 2 and 3; (d) phase difference between vibrators 3 and 4 .

TABLE 3: The stable phase differences for $\eta_{1}=\eta_{2}=1, \eta_{3}=0.75, \eta_{4}=0.5$.

\begin{tabular}{ccccc}
\hline & & \multicolumn{2}{c}{ Simulation of point A } & \multicolumn{2}{c}{$\begin{array}{c}\text { Characteristic analysis* } \\
\text { Phase differences }\end{array}$} \\
\hline $2 \alpha_{1}$ & $1.37^{\circ}$ & $1.31^{\circ}$ & $13.9^{\circ}$ & $6^{\circ}$ \\
$2 \alpha_{2}$ & $-2.71^{\circ}$ & $-2.78^{\circ}$ & $-15.5^{\circ}$ & $3^{\circ}$ \\
$2 \alpha_{3}$ & $-8.11^{\circ}$ & $-8.13^{\circ}$ & $-9.18^{\circ}$ & $0^{\circ}$ \\
\hline
\end{tabular}

*The results of characteristic analysis can be observed in Figure 4(c).

5.2. Subresonant Region (Simulation Point A) Simulations. The analysis method in this section is the same as the previous section, and the results will be presented in the table form.

5.2.1. Simulation for $\eta_{1}=\eta_{2}=\eta_{3}=\eta_{4}=1$. The stable phase differences for Figures $8(\mathrm{~b})-8(\mathrm{~d})$ are shown in Table 1.

The stable phase differences of simulation and characteristic analysis are in good agreement with each other. In Figures $8(\mathrm{e})$ and $8(\mathrm{f})$, the resonant responses of the rigid frame in $x$ and $y$ directions are larger and stabilize eventually in the range of $-15 \sim+15$, while the resonant response in $\psi$ direction is zero. The above results indicate that the exciting forces superimpose positively, and a strong vibration occurs in the rigid frame, when the system operates in the subresonant region.

5.2.2. Simulation for $\eta_{1}=\eta_{2}=\eta_{3}=1, \eta_{4}=0.5$. As shown in Figure 9, the results are given in Table 2.

5.2.3. Simulation for $\eta_{1}=\eta_{2}=1, \eta_{3}=0.75, \eta_{4}=0.5$. From Figure 10, the results can be obtained and are presented in Table 3.

We can observe that the phase differences before disturbance are approximately equal to those after disturbance.
The phenomenon accords with that of the characteristic analysis.

\section{Conclusions}

According to the above theoretical derivation, numerical characteristic analysis, and simulations, the following conclusions are given:

(1) Through contrasting simulations of two regions in different $\eta$, we can observe the phase differences in $\eta_{1}=\eta_{2}=\eta_{3}=\eta_{4}=1$ are more stable, which indicates that structure parameters have a large effect on synchronization, i.e., the better the symmetry of the system is, the more easily the vibrators achieve synchronous stability.

(2) Resonant regions of the vibrating system are eventually divided to two regions: subresonant and superresonant; the phase differences in the subresonant maintain around $0^{\circ}$ and those in the superresonant maintain around $90^{\circ}$ and $180^{\circ}$. The resonant responses of the rigid frame with double resonant types in $x, y$, and $\psi$ directions are also discussed. These results indicate that the exciting forces of the system can be superposed positively, and the rigid frame has a large vibration amplitude, when the working region point of the vibrating 
system is in the subresonant. While in the superresonant, the phase differences among vibrators cannot be stabilized in the vicinity of zero, so it causes exciting forces to counteract each other, and the rigid frame produces no vibration.

(3) Utilizing the present study results of the synchronization and stability in the superresonant states, we can design a new type of high-frequency vibrating miller in engineering. For example, using the model and parameters of this paper, a new vibrating ball mill with four rollers can be designed, and the working region of the system is selected to be the superresonant region. Under the condition of ensuring good vibrating ball mill technology, the system can also reach the effect of vibration isolation automatically and has little harmful effect on the surrounding area.

\section{Data Availability}

The data used to support the findings of this study are available from the corresponding author upon request.

\section{Conflicts of Interest}

The authors declare that there are no conflicts of interest regarding the publication of this paper.

\section{Acknowledgments}

This study was funded by the National Natural Science Foundations of China (51675090), China Postdoctoral Science Foundation (2017M621145), and Fundamental Research Funds for the Central Universities (N170304013).

\section{References}

[1] J. Rayleigh, Theory of Sound, Dover, New York, NY, USA, 1945.

[2] B. van der Pol, "Theory of the amplitude of free and forced triode vibration," Radio Review, vol. 1, pp. 701-710, 1920.

[3] C. Huygens, Horologium Oscilatorium, Paris, French, 1673.

[4] L. M. Pecora, T. L. Carroll, G. A. Johnson, D. J. Mar, and J. F. Heagy, "Fundamentals of synchronization in chaotic systems, concepts, and applications," Chaos: An Interdisciplinary Journal of Nonlinear Science, vol. 7, no. 4, pp. 520-543, 1997.

[5] G. V. Osipov, O. I. Kanakov, C. K. Chan et al., "Synchronization phenomena in networks of oscillatory and excitable Luo-Rudy cells," in Complex Dynamics in Physiological Systems: From Heart to Brain. Understanding Complex Systems, pp. 107-126, Springer Complexity, Berlin, Germany, 2009.

[6] A. Teufel, A. Steindl, and H. Troger, "Synchronization of two flow excited pendula," Communications in Nonlinear Science and Numerical Simulation, vol. 11, no. 5, pp. 577-594, 2006.

[7] M. Kapitaniak, K. Czolczynski, P. Perlikowski, A. Stefanski, and T. Kapitaniak, "Synchronous states of slowly rotating pendula," Physics Reports, vol. 541, no. 1, pp. 1-44, 2014.

[8] Q. Lu, H. Gu, Z. Yang, X. Shi, L. Duan, and Y. Zheng, "Dynamics of firing patterns, synchronization and resonances in neuronal electrical activities: experiments and analysis," Acta Mechanica Sinica, vol. 24, no. 6, pp. 593-628, 2008.

[9] T. Yan, L. Yang, B. Yan, W. Zhou, L. Chen, and W. Zhou, "Dynamic shift coordinated control based on motor active speed synchronization with the new hybrid system," Shock and Vibration, vol. 2017, Article ID 2716251, 16 pages, 2017.

[10] Q. Han, M. Wang, and H. Chu, "Nonsynchronous vibrations of rotor system with an oil-block inside the rotating drum," Advances in Vibration Engineering, vol. 12, no. 2, pp. 165-178, 2013.

[11] A. Prasad, "Universal occurrence of mixed-synchronization in counter-rotating nonlinear coupled oscillators," Chaos, Solitons \& Fractals, vol. 43, no. 1-12, pp. 42-46, 2010.

[12] E. Jiaqiang, C. Qian, T. Liu, and G. Liu, "Chaos analysis on the acceleration control signals of the piezoelectric actuators in the stewart platform," Shock and Vibration, vol. 2016, Article ID 8087176, 9 pages, 2016.

[13] M. Mobaraki, R. S. Semken, A. Mikkola, and J. Pyrhönen, "Enhanced sludge dewatering based on the application of high-power ultrasonic vibration," Ultrasonics, vol. 84, pp. 438-439, 2018.

[14] I. I. Blekhman, Synchronization in Science and Technology, ASME Press, New York, NY, USA, 1988.

[15] I. I. Blekhman and V. S. Sorokin, "On the separation of fast and slow motions in mechanical systems with high-frequency modulation of the dissipation coefficient," Journal of Sound and Vibration, vol. 329, no. 23, pp. 4936-4949, 2010.

[16] I. I. Blekhman, A. L. Fradkov, H. Nijmeijer, and A. Y. Pogromsky, "On self-synchronization and controlled synchronization," Systems \& Control Letters, vol. 31, no. 5, pp. 299-305, 1997.

[17] J. Inoue and Y. Araki, "Self-synchronization of mechanical system (multiple cycle)," Proceedings of Japanese Mechanical Engineering Society, vol. 42, pp. 103-110, 1981, in Japanese.

[18] D. Quinn, R. Rand, and J. Bridge, "The dynamics of resonant capture," Advances in Nonlinear Dynamics: Methods and Applications, vol. 8, pp. 1-20, 1995.

[19] B. C. Wen, H. Zhang, S. Y. Liu et al., Theory and Techniques of Vibrating Machinery and Their Applications, Science Press, Beijing, China, 2010.

[20] B. C. Wen, J. Fan, C. Y. Zhao et al., Vibration Synchronization and Controlled Synchronization in Engineering, Science Press, Beijing, China, 2009.

[21] P. Fang and Y. J. Hou, "Synchronization characteristics of a rotor-pendula system in multiple coupling resonant systems," Proceedings of the Institution of Mechanical Engineers, Part C: Journal of Mechanical Engineering Science, vol. 232, no. 10, pp. 1802-1822, 2017.

[22] J. M. Balthazar, J. L. P. Felix, and R. M. Brasil, "Short comments on self-synchronization of two non-ideal sources supported by a flexible portal frame structure," Modal Analysis, vol. 10, no. 12, pp. 1739-1748, 2004.

[23] J. M. Balthazar, J. L. P. Felix, and R. M. Brasil, "Some comments on the numerical simulation of self-synchronization of four non-ideal exciters," Applied Mathematics and Computation, vol. 164, no. 2, pp. 615-625, 2005.

[24] X. L. Zhang, B. C. Wen, and C. Y. Zhao, "Vibratory synchronization transmission of a cylindrical roller in a vibrating mechanical system excited by two exciters," Mechanical Systems and Signal Processing, vol. 96, pp. 88-103, 2017.

[25] X. L. Zhang, C. Y. Zhao, and B. C. Wen, "Theoretical and experimental study on synchronization of the two homodromy exciters in a non-resonant vibrating system," Shock and Vibration, vol. 20, no. 2, pp. 327-340, 2013. 
[26] X. L. Zhang, B. C. Wen, and C. Y. Zhao, "Synchronization of three non-identical coupled exciters with the same rotating directions in a far-resonant vibrating system," Journal of Sound and Vibration, vol. 332, no. 9, pp. 2300-2317, 2013.

[27] C. Y. Zhao, H. T. Zhu, R. Z. Wang, and B. C. Wen, "Synchronization of two non-identical coupled exciters in a nonresonant vibrating system of linear motion. Part I: theoretical analysis," Shock and Vibration, vol. 16, no. 5, pp. 505-516, 2009.

[28] Y. Yang, R. Fu, and L. Huang, "Robust analysis and synthesis for a class of uncertain nonlinear systems with multiple equilibria," Systems \& Control Letters, vol. 53, no. 2, pp. 89-105, 2004. 


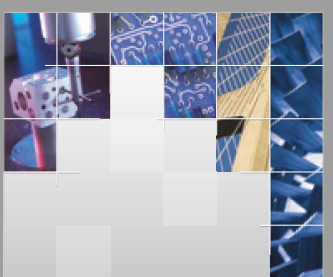

\section{Enfincering}
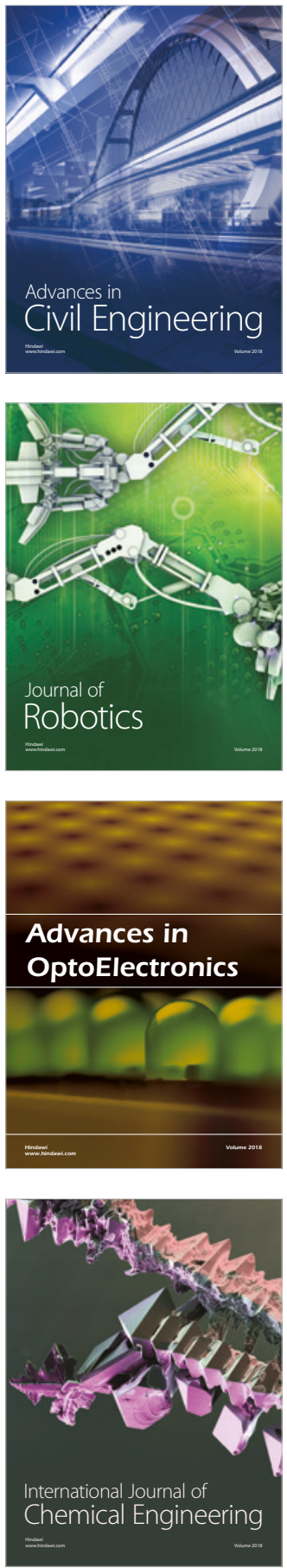

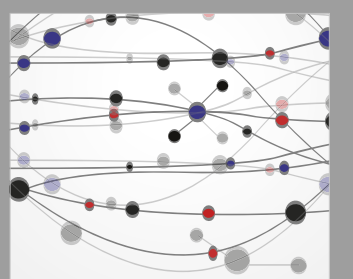

\section{Rotating \\ Machinery}

The Scientific World Journal

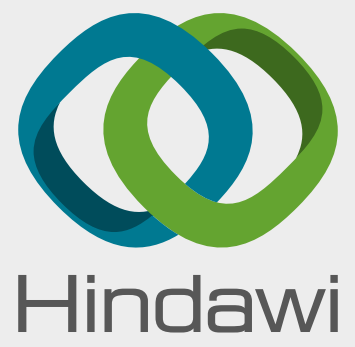

Submit your manuscripts at

www.hindawi.com
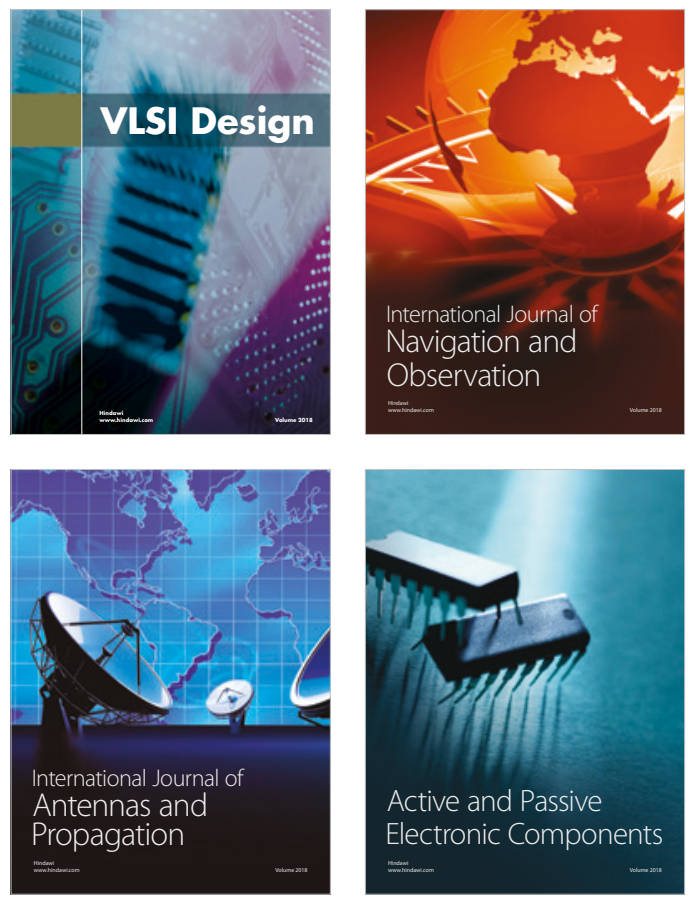
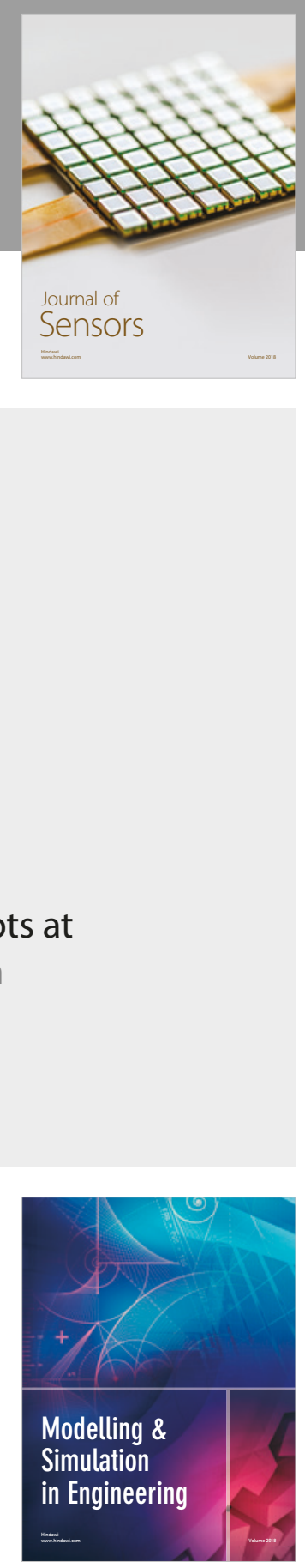

\section{Advances \\ Multimedia}
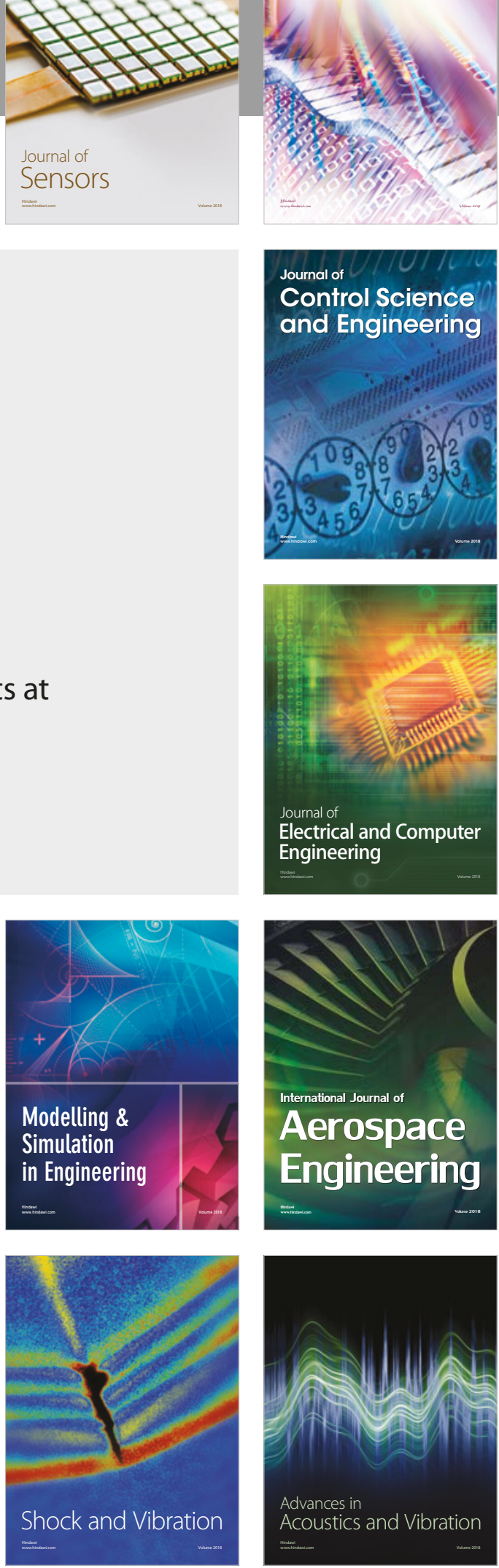